ARTICLE

\title{
Functional role of Tet-mediated RNA hydroxymethylcytosine in mouse ES cells and during differentiation
}

Jie Lan ${ }^{1,9}$, Nicholas Rajan ${ }^{1,9}$, Martin Bizet ${ }^{1}$, Audrey Penning (10 1, Nitesh K. Singh ${ }^{1}$, Diana Guallar (D) ${ }^{2}$, Emilie Calonne ${ }^{1}$, Andrea Li Greci, Elise Bonvin', Rachel Deplus', Phillip J. Hsu ${ }^{3}$, Sigrid Nachtergaele ${ }^{3}$, Chengjie $\mathrm{Ma}^{4}$, Renhua Song (10 ${ }^{5}$, Alejandro Fuentes-Iglesias (10 ${ }^{2}$, Bouchra Hassabi ${ }^{1}$, Pascale Putmans ${ }^{1}$, Frédérique Mies ${ }^{1}$, Gerben Menschaert ${ }^{6}$, Justin J. L. Wong (1) ${ }^{5}$, Jianlong Wang (1) ${ }^{7}$, Miguel Fidalgo (i) ${ }^{2}$, Bifeng Yuan (i) ${ }^{4} \&$ François Fuks (1) ${ }^{1,8 \times}$

Tet-enzyme-mediated 5-hydroxymethylation of cytosines in DNA plays a crucial role in mouse embryonic stem cells (ESCs). In RNA also, 5-hydroxymethylcytosine (5hmC) has recently been evidenced, but its physiological roles are still largely unknown. Here we show the contribution and function of this mark in mouse ESCs and differentiating embryoid bodies. Transcriptome-wide mapping in ESCs reveals hundreds of messenger RNAs marked by $5 \mathrm{hmC}$ at sites characterized by a defined unique consensus sequence and particular features. During differentiation a large number of transcripts, including many encoding key pluripotency-related factors (such as Eed and Jarid2), show decreased cytosine hydroxymethylation. Using Tet-knockout ESCs, we find Tet enzymes to be partly responsible for deposition of $5 \mathrm{hmC}$ in mRNA. A transcriptome-wide search further reveals mRNA targets to which Tet1 and Tet2 bind, at sites showing a topology similar to that of $5 \mathrm{hmC}$ sites. Tetmediated RNA hydroxymethylation is found to reduce the stability of crucial pluripotencypromoting transcripts. We propose that RNA cytosine 5-hydroxymethylation by Tets is a mark of transcriptome flexibility, inextricably linked to the balance between pluripotency and lineage commitment.

\footnotetext{
${ }^{1}$ Laboratory of Cancer Epigenetics, Faculty of Medicine, ULB Cancer Research Center (U-CRC), Welbio Investigator, Université Libre de Bruxelles (ULB), Brussels, Belgium. ${ }^{2}$ CiMUS, Universidade de Santiago de Compostela-Health Research Institute (IDIS), Santiago de Compostela, Coruña, Spain. ${ }^{3}$ Department of Chemistry, Department of Biochemistry and Molecular Biology, Institute for Biophysical Dynamics, and Howard Hughes Medical Institute, University of Chicago, Chicago, IL 60637, USA. ${ }^{4}$ Key Laboratory of Analytical Chemistry for Biology and Medicine (Ministry of Education), Department of Chemistry, Wuhan University, 430072 Wuhan, People's Republic of China. ${ }^{5}$ Epigenetics and RNA Biology Program Centenary Institute, The University of Sydney, Camperdown, NSW 2050, Australia. ${ }^{6}$ Department of Mathematical Modeling, Statistics and Bioinformatics, Faculty of Bioscience Engineering, Lab of Bioinformatics and Computational Genomics, Ghent University, Ghent, Belgium. ${ }^{7}$ Department of Medicine, Columbia Center for Human Development (CCHD), Columbia University Irving Medical Center (CUIMC), New York, NY 10032, USA. ${ }^{8}$ WELBIO (Walloon Excellence in Lifesciences \& Biotechnology), Brussels, Belgium. ${ }^{9}$ These authors contributed equally: Jie Lan, Nicholas Rajan. ${ }^{{ }^{2}}$ email: ffuks@ulb.ac.be
} 
n DNA, the family of TET methyldioxygenases (TET1, TET2, and TET3) is known to catalyze hydroxylation of 5methylcytosine to generate 5-hydroxymethylcytosine ${ }^{1,2}$. This reaction, which requires $\mathrm{Fe}^{2+}$ and a-ketoglutarate as co-factors, adds an additional layer of complexity to the epigenetic regulation of DNA methylation, as it can act as an intermediate in DNA demethylation pathways ${ }^{2-4}$. Recent advances have provided a more precise picture of the roles of TET-mediated DNA hydroxymethylation in several diseases such as cancer ${ }^{5-9}$ and in various biological contexts ${ }^{10-12}$. Notably, it is increasingly clear that DNA hydroxymethylation has a role in key physiological processes, including pre-implantation ${ }^{13-15}$, ESC pluripotency, and differentiation ${ }^{16-22}$. For example, TET triple knockout (TKO) and single TET KO studies reveal that while TET proteins are not required for ESC maintenance, they are essential for the proper differentiation capacity and the generation of functional embryonic structures $20,21,23$.

Previous work showed that Tet-mediated 5-hydroxymethylation $(5 \mathrm{hmC})$ occurs also in RNA context ${ }^{24-30}$, but in RNA its roles are just beginning to be appreciated. Tet-deficient Drosophila fruitflies suffer impaired brain development, accompanied by decreased RNA hydroxymethylation ${ }^{28}$. In mammals, Tet 2 acts via $5 \mathrm{hmC}$ marking of RNA to promote pathogen infectioninduced myelopoiesis through mRNA oxidation ${ }^{29}$ and to control endogenous retroviruses (ERVs) ${ }^{30}$. Tet-mediated RNA hydroxymethylation has also been reported to occur in $\mathrm{ESCs}^{24}$. However, to date, the distribution, function, and biological relevance of $5 \mathrm{hmC}$ remain unknown.

Here, we show that Tet enzymes are required for deposition of $5 \mathrm{hmC}$ in mRNAs, and notably in key pluripotency-related transcripts. Interestingly, we find that during differentiation, a large number of these transcripts have a reduced level of cytosine hydroxymethylation. We report that $5 \mathrm{hmC}$ reduces the stability of important pluripotency-promoting transcripts, and propose Tet-mediated RNA hydroxymethylation as an additional level of regulation of the ESC self-renewal network.

\section{Results}

Transcriptome-wide distribution of $5 \mathrm{hmC}$ in ESCs and EBs. We initiated our study by assessing the transcriptome-wide $5 \mathrm{hmC}$ landscape in mouse ESCs. For this we used our previously described hMeRIP-Seq method ${ }^{28}$, involving immunoprecipitation of $5 \mathrm{hmC}$-containing RNA with an anti-5hmC antibody (Fig. 1a and Supplementary Fig. 1a), followed by next-generation sequencing (see Methods for experimental details and validation of antibody specificity). This approach revealed a total of 1633 peaks ( $q$-values $<0.05$ ) in 795 transcripts (Fig. $1 b, c$ and Supplementary Data 1). The top hydroxymethylated mRNA targets are shown in Supplementary Fig. 1b and examples of enrichment profiles with the corresponding input tracks are shown in Fig. 1c (additional examples are shown in Supplementary Fig. 1c). We observed a non-random distribution of $5 \mathrm{hmC}$, the mark occurring mostly in introns (Fig. 1d). Subsequent analyses revealed a specific UC-rich motif at peak centers (Fig. 1e and Supplementary Fig. 1d), consistently with findings of our previous study on Drosophila S2 cells ${ }^{28}$.

To see whether transcripts relevant to ESC pluripotency might be present among those identified here, we compared the abovementioned hMeRIP-Seq data sets with publicly available mouse ESC data sets defining signatures for the regulatory circuitry controlling the embryonic stem cell state ${ }^{31-35}$. Of the $7955 \mathrm{hmC}$ modified transcripts identified, 110 were found to encode pluripotency-related factors, including key ESC pluripotency regulators such as Eed, Jarid2, Smarcc1, Paf1, and Mbd3 (Fig. If and Supplementary Fig. 1e and Supplementary Data 1). We observed the same features of non-random distribution of $5 \mathrm{hmC}$ peaks within these pluripotency-related transcripts, with the mark occurring mostly in introns (Supplementary Fig. 1f). This transcriptome-wide assessment of $5 \mathrm{hmC}$ in WT mouse ESCs thus highlights a unique distribution and features of $5 \mathrm{hmC}$ sites in hundreds of transcripts, notably of many key pluripotencyrelated mRNAs.

The above-mentioned strong $5 \mathrm{hmC}$ enrichment within introns (cf. Fig. 1d and Supplementary Fig. 1f) prompted us to assess the level of $5 \mathrm{hmC}$ by dot blotting on the three following RNA fractions: nascent chromatin-associated, nucleoplasmic, and cytoplasmic. As shown in Supplementary Fig. 1g, we observed that chromatin-associated RNAs were readily hydroxymethylated. These data suggest enrichment in $5 \mathrm{hmC}$ of intronic regions of unspliced nascent pre-mRNAs. We also evaluated the role of Tet-mediated hydroxymethylation in splicing regulation, by means of paired-end RNA-Seq in WT and TKO ESCs, followed by differential splicing analysis. We found Tet-mediated hydroxymethylation to be associated with a higher ratio of spliced to unspliced transcripts (Supplementary Fig. 1h, i and Supplementary Data 2).

We next examined how the level and distribution of $5 \mathrm{hmC}$ might change during mESC differentiation to embryoid bodies (EBs). We used conditions for spontaneous differentiation of ESCs to EBs at an early time (day 4), which allowed us to focus on the role played by Tet 1 and Tet 2 at an early stage of ESC differentiation. In agreement with a previous report ${ }^{36}$, transcriptlevel expression of Tet 1 and Tet2, as measured by RT-qPCR, were decreased upon ESC-to-EB differentiation, while Tet3 was still barely expressed (Fig. 1g). Proper differentiation of ESCs to EBs was checked by quantifying markers of pluripotency and early differentiation (Supplementary Fig. 1j). We first assessed the global $5 \mathrm{hmC}$ level by dot blotting applied to RNA extracts. EBs displayed a lower $5 \mathrm{hmC}$ signal than ESCs (Fig. $1 \mathrm{~h}$ and Supplementary Fig. 1k). We then performed hMeRIP-Seq on ESCs and EBs. As shown in Fig. 1i, 5hmC marking was found to decrease in over $80 \%$ of the transcripts upon ESC-to-EB differentiation. The observed $5 \mathrm{hmC}$ changes were widely distributed within transcripts (Supplementary Fig. 11 and Supplementary Data 3). Of the 649 mRNAs showing reduced $5 \mathrm{hmC}$ in EBs vs ESCs, 72 encode pluripotency-promoting factors, e.g., Eed, Jarid2, and Dab1 (Fig. 1i, j and Supplementary Fig. 1m). ESC-to-EB differentiation thus leads, concomitantly with reduced Tet 1 and Tet 2 expression, to a marked decrease in $5 \mathrm{hmC}$, notably affecting key pluripotency-related mRNAs.

Tet-mediated hydroxymethylation in ESCs. What is the contribution of Tet proteins to mRNA hydroxymethylation in ESCs? To answer this question, we used previously generated Tet1/2/3 triple knockout (TKO) mouse ESCs ${ }^{21}$. In line with the previous work $^{24}$, TKO ESCs showed a substantially lower ( $\sim 50 \%$ lower) global $5 \mathrm{hmC}$ level than WT ESCs, as measured by dot blotting and mass spectrometry (Fig. 2a and Supplementary Fig. 2a). It is noteworthy that $\mathrm{m} 5 \mathrm{C}$ remained at a similar level in TKO cells (Supplementary Fig. 2b), consistently with previously published data. Since Vitamin C is a known cofactor for Tet-mediated DNA hydroxymethylation in $\mathrm{ESCs}^{4}$ (Supplementary Fig. 2c), we tested whether Vitamin C might also induce Tet-dependent RNA hydroxymethylation. This proved to be the case: dot blotting applied to RNA from WT and TKO ESCs revealed, upon Vitamin $\mathrm{C}$ treatment, a rise in the global level of $5 \mathrm{hmC}$ in the WT cells only (Supplementary Fig. 2d).

To see which Tets might be responsible for $5 \mathrm{hmC}$ marking, we first measured the global $5 \mathrm{hmC}$ level in Tet1/2/3 triple knockout $(\mathrm{TKO})^{21}$, Tet $1 / 2$ double knockout $(\mathrm{DKO})^{20}$, and Tet3 knockout 
a

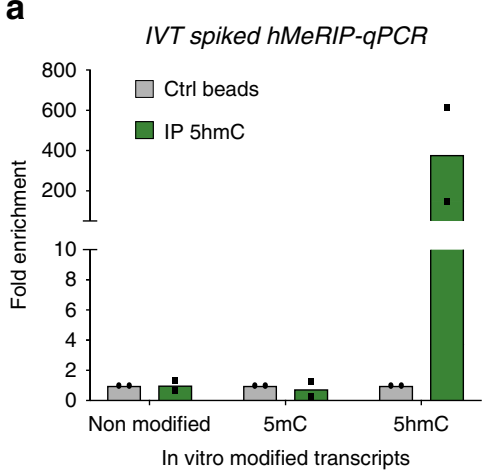

d

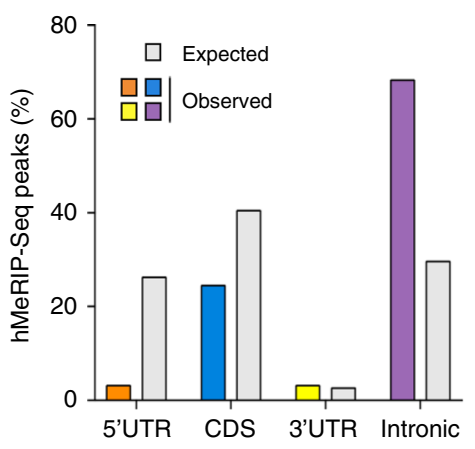

h

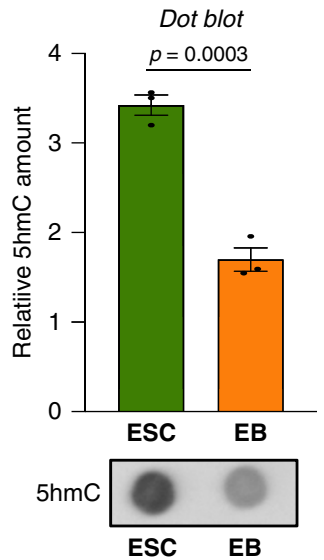

b

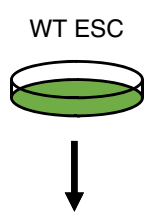

hMeRIP-Seq

\begin{tabular}{|l|l|}
\hline Peaks & 1633 \\
\hline Transcripts & 795 \\
\hline
\end{tabular}

e

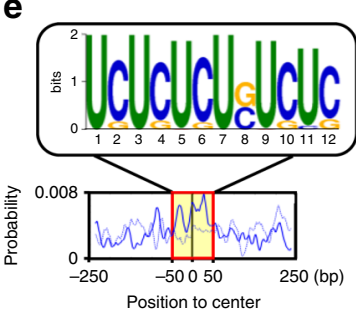

f

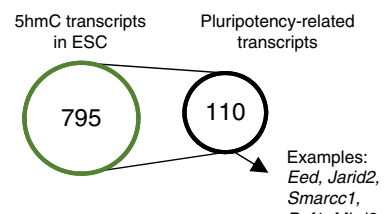

C
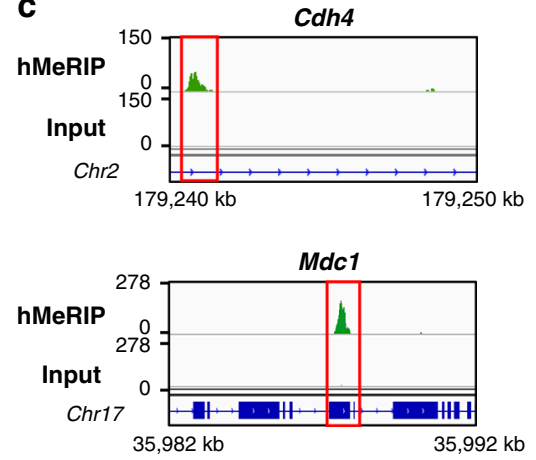

g

Spontaneous differentiation
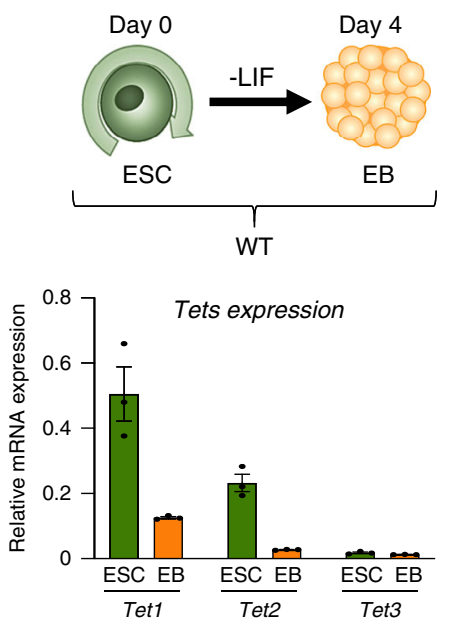

i

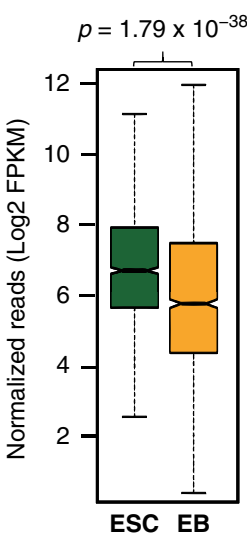

hMeRIP-Seq

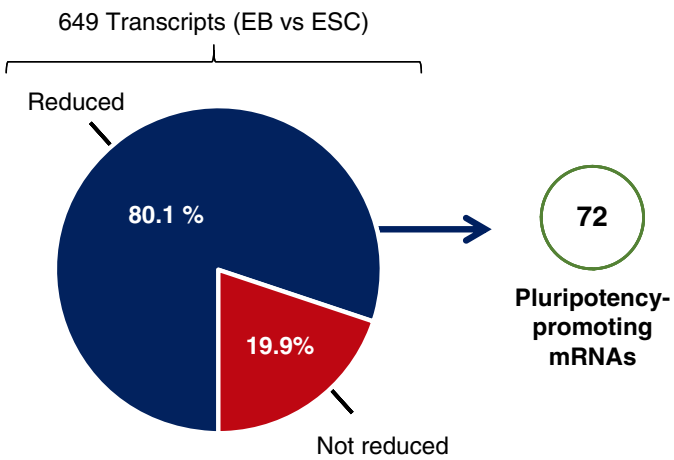

ESCs $^{37}$. Dot blots for Tet1- and Tet2-depleted ESCs (DKO) showed a reduction of the $5 \mathrm{hmC}$ level similar to that observed for TKO ESCs, while dot blots for Tet $3 \mathrm{KO}$ ESCs showed no decrease in global 5hmC (Supplementary Fig. 2a). These results indicate that while Tet1, Tet2, or both are involved in $5 \mathrm{hmC}$ marking of mRNAs in ESCs, this seems not to be the case for Tet3.
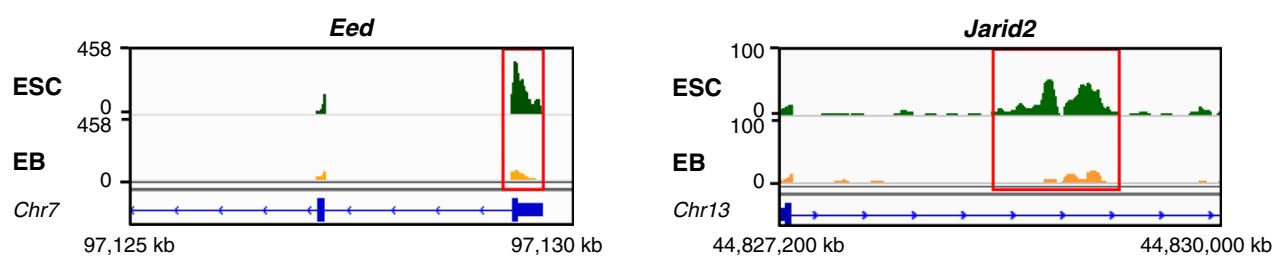

To examine the contribution of Tets to $5 \mathrm{hmC}$ marking at the transcript level, we performed hMeRIP-Seq on WT and TKO ESCs. As shown in Fig. 2b, we observed a significant reduction of 5 hmC reads $\left(P<10^{-34}\right)$ in TKO as compared to WT ESCs. In Tet-depleted ESCs, $68.1 \%$ of the mRNAs (575 transcripts) showed a reduced $5 \mathrm{hmC}$ level (Supplementary Data 4), among 
Fig. 1 Transcriptome-wide distribution of $\mathbf{5 h m C}$ in ESCs and EBs. a Specificity of 5hmC antibody. Only the 5hmC-modified transcript shows enrichment after hMeRIP compared to the controls. Unmodified, 5mC-modified, and 5hmC-modified transcripts (IVT: in vitro transcribed) were used to spike total RNA prior to hMeRIP-qPCR. Data are means \pm SEM ( $n=2$ independent experiments). b hMeRIP-Seq in WT ESCs reveals the presence of $5 \mathrm{hmC}$ within many transcripts $(n=3)$. Experiments were performed in biological triplicate and results were normalized as described in the "Methods" section. c Exemplative hMeRIP-Seq profiles of Cdh4 and Mdc1 in WT ESCs with their corresponding input control tracks (IGV tracks) (red frame shows peak location). d Bar chart showing the distribution of $5 \mathrm{hmC}$ peaks according to the type of structural element within transcripts, next to the expected distribution. e Top sequence motif identified in the centers of $5 \mathrm{hmC}$ peaks ( $E$-value $<2.2 \mathrm{e}-117$ ). $\mathbf{f} 5 \mathrm{hmC}$ is found in many key pluripotency-related mRNAs. Comparison of the above hMeRIP-Seq data sets with publicly available mouse ESC data sets ${ }^{10-14}$, with representative examples of known transcripts encoding ESC core pluripotency regulators such as Eed, Jarid2, Smarcc1, Paf1, and Mbd3. g Scheme of the previously reported protocol ${ }^{36}$ used for spontaneous differentiation of ESCS into EBs upon LIF removal, as described in the Methods section, with their relative Tet expression levels as measured by RT-qPCR. Data are means \pm SEM ( $n=3$ independent experiments). $\mathbf{h}$ Decreased global $5 \mathrm{hmC}$ during spontaneous differentiation, as assessed by dot blotting. Data are means \pm SEM (representative blot from three independent experiments, two-tailed Student's t-test). i Many pluripotency-related transcripts show reduced 5hmC during differentiation. Left: Box plot for hMeRIP-Seq ESCs and EBs ( $n=3$ independent experiments, two-tailed Student's $t$-test). In the box plot, the boxes represent the interquartile range of the records, and the lines across the boxes indicate the median value of the records. The whiskers indicate the highest and lowest values among the records that are no more than 1.5 times greater than the interquartile range. The range between notches represents the $95 \%$ confidence interval. Right: Pie chart highlighting the percentage of transcripts showing reduced 5 hmC marking, 72 of which are identified as pluripotency-promoting mRNAs. j Exemplative hMeRIP-Seq profiles of Eed and Jarid2 in ESCs vs EBs (IGV tracks) (red frame shows peak location). Source data are provided as a Source Data File.

which 52 are known to encode critical pluripotency factors such as Eed, Dab1, and Sfpq (Fig. 2c, d and Supplementary Fig. 2e). In mRNAs showing a reduced $5 \mathrm{hmC}$ level in TKO ESCs, we found an overrepresented UC-rich motif (Fig. 2e) highly similar to the $5 \mathrm{hmC}$ site identified in WT ESCs (cf. Fig. 1e). Furthermore, the $5 \mathrm{hmC}$ decrease was found to occur mostly in introns (Fig. 2f). Together, these results indicate that Tets are required for deposition of $5 \mathrm{hmC}$ in mRNAs, and notably in key pluripotency-related transcripts. Given that Tets knockout did not totally abolish $5 \mathrm{hmC}$ marking either globally or at the transcript level, it seems likely that additional enzymes and/or chemical processes contribute to mRNA hydroxymethylation in ESCs. To test whether $5 \mathrm{hmC}$ in mRNA might form through other chemical processes, we specifically evaluated whether $5 \mathrm{hmC}$ might be induced by cellular reactive oxygen species (ROS). This seems not to be the case, as treatment of ESCs with either buthionine sulfoximide (BSO) or $\mathrm{H}_{2} \mathrm{O}_{2}$ did not change the global level of $5 \mathrm{hmC}$ in mRNA (Supplementary Fig. 2f).

Tet1- and Tet2-bound mRNAs in ESCs. As the mRNA targets of Tets are unknown, we next sought to identify these targets throughout the transcriptome by generating CRISPR knock-in ESCs for Tet 1 and Tet2. Using the CRISPR genome-editing tool in ESCs, we added a Flag-tag to endogenous Tet1 or Tet2. RNA immunoprecipitation (RIP) with anti-Flag antibody was then performed, followed by deep sequencing (Fig. 3a and Supplementary Fig. 3a). As shown in Fig. 3b, RIP-Seq for endogenous Tet1 identified 7798 bound targets. Similar experiments for Tet2 revealed its binding to 6659 transcripts (Fig. 3c and Supplementary Data 5). Interestingly, an RNA-binding domain (RBD) within Tet2 has recently been identified by proteomic approach and is a sequence adjacent to the C-terminal catalytic domain ${ }^{38}$. Exploiting this finding, we used CRISPR-Cas9 to delete from endogenous Tet2 the 54 amino acids corresponding to the whole sequence encoding the identified site (Fig. 3a and Supplementary Fig. 3a). The corresponding knock-in cells thus produced a Flagtagged Tet2 protein, either WT or deleted of the RBD (Tet $2 \triangle \mathrm{RBD})$. RIP-Seq for Tet $2 \triangle \mathrm{RBD}$ revealed that about $30 \%$ of Tet2 targets are dependent on its RBD (Fig. 3c-e). The identified RBD thus contributes at least partly to specific Tet 2 targeting. A comparison of the Tet1- and Tet2-RIP-Seq data revealed considerable and significant overlap between Tet2- and Tet1-bound targets, corresponding to $78.7 \%$ of the Tet2-bound transcripts (Fig. 3f). We also compared our RIP-Seq data for Tet1 and Tet2 with the hMeRIP-Seq data. Although many Tet1- and Tet2interacting transcripts seemed not to be hydroxymethylated, $64.5 \%$ of the identified hydroxymethylation targets appeared to interact with Tet1 and/or Tet2 (Fig. 3g). We found that when Tet 1 and Tet 2 are bound to $5 \mathrm{hmC}$ targets, they are mostly bound together, rather than alone (Fig. 3g). This suggests that both Tet1 and Tet 2 contribute to $5 \mathrm{hmC}$ and that they have redundant roles in mRNA hydroxymethylation in ESCs. We further found Tet1 and Tet2, like $5 \mathrm{hmC}$, to associate preferentially with intronic regions (Supplementary Fig. 3b). Likewise, within 5hmC-enriched sites, Tet 1 and Tet2 appeared to bind targets preferentially characterized by a UC-rich motif (Supplementary Fig. 3c). Interestingly, the percentage of pluripotency-related transcripts showing both enrichment in 5hmC and binding to Tet1 and/or Tet2 was particularly high, i.e., 70\% (Supplementary Fig. 3d). These transcripts notably included Eed, Jarid2, Smarcc1, and Dab1. It is worth mentioning that in addition to binding to 5hmC-modified targets, Tet1 and Tet2 also bound to many unmodified transcripts. Using publicly available data ${ }^{19}$ we observed, upon comparing Tet1/2-bound 5hmC-modified and unmodified RNAs, a lower level of 5-methylcytosine in genes bodies related to unmodified RNAs than in genes related to 5hmC-modified ones (Supplementary Fig. 3e). This suggests potential catalysis-independent roles for Tet1 and Tet2.

To further investigate the effect of Tet $1 / 2$ binding on $5 \mathrm{hmC}$ modified and unmodified mRNAs, we performed RNA-Seq experiments on TKO ESCs and analyzed upregulated and downregulated transcripts upon Tet depletion. Firstly, by comparing $5 \mathrm{hmC}$ targets from hMeRIP-Seq with Tet-regulated transcripts, we found $55.6 \%$ of the $5 \mathrm{hmC}$-enriched targets to be upregulated and $44.4 \%$ to be downregulated (Supplementary Fig. 3f). Secondly, a comparison of Tet1/Tet2-bound mRNAs from RIP-Seq with RNA-Seq in TKO ESCs showed both upregulated (65.9\%) and downregulated transcripts (34.1\%) (Supplementary Fig. 3g). Lastly, we also looked at the overlap between $5 \mathrm{hmC}$-enriched targets bound by Tet1/2 and up- or downregulated transcripts. We found a significant number of downregulated transcripts harboring $5 \mathrm{hmC}$ to be bound by Tet1/ $2(68.2 \%)$. Many upregulated transcripts enriched in $5 \mathrm{hmC}$ were also found to interact with Tet1/2 (67.3\%) (Supplementary Fig. 3h).

This transcriptome-wide investigation thus shows that a large number of transcripts are bound by Tet1, Tet2, or both. We observed that the majority of $5 \mathrm{hmC}$ targets are bound by Tet $1 / 2$, 
a

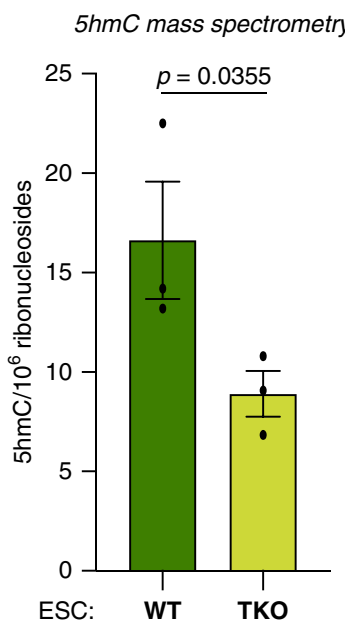

c

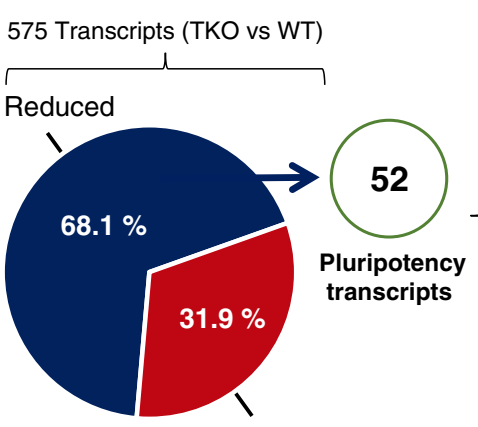

Not reduced b

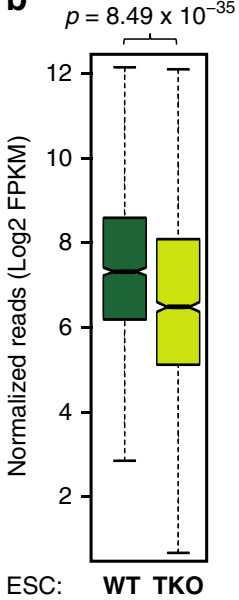

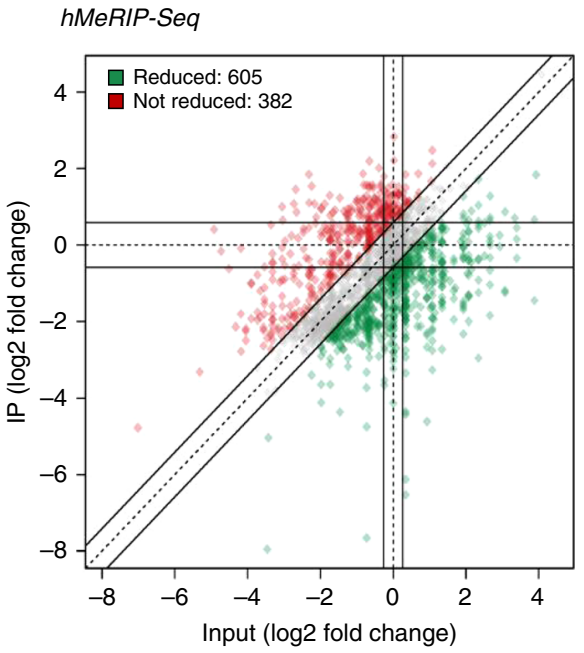

d

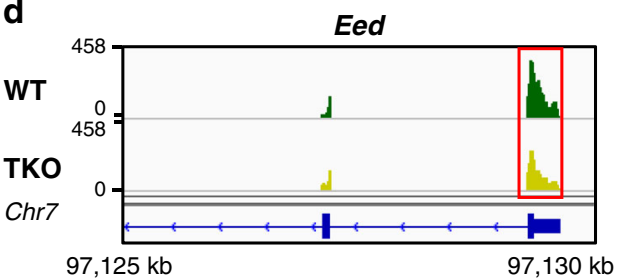

Dab1

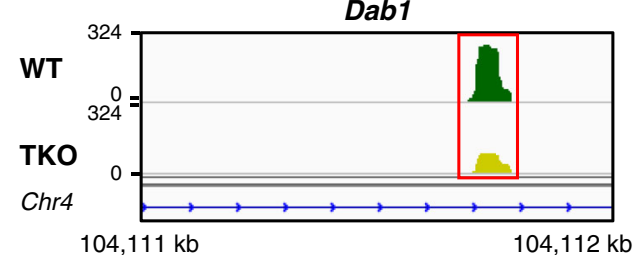

e
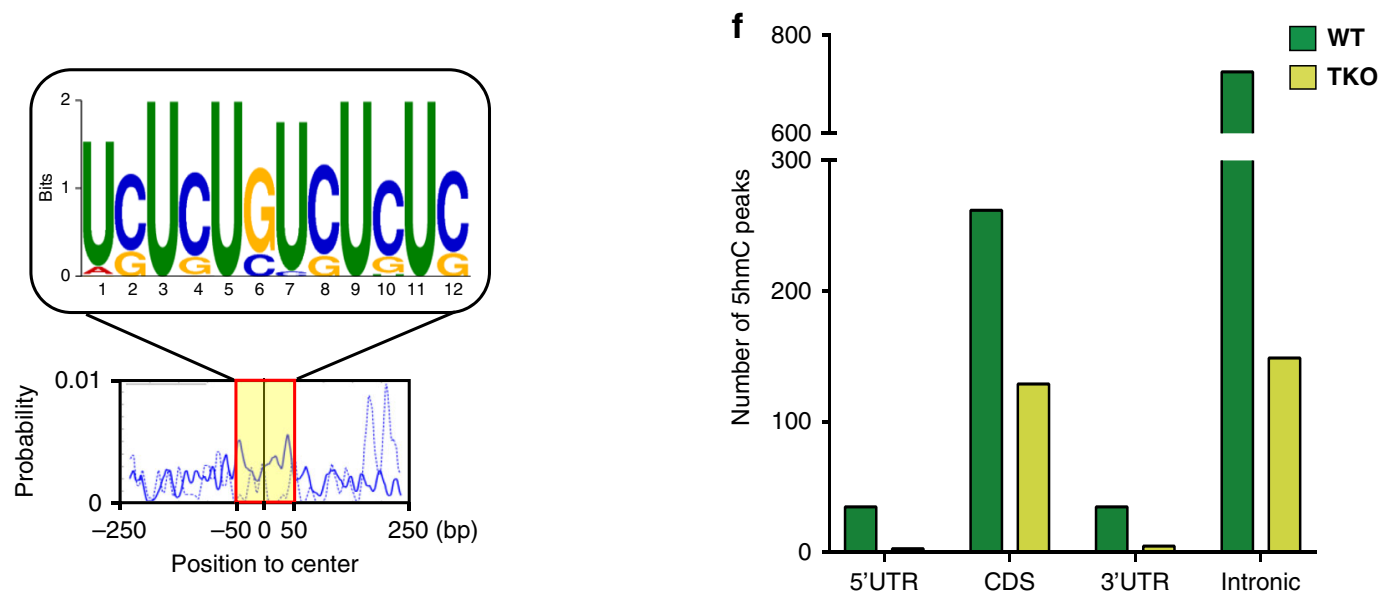

Fig. 2 Tets are required for $\mathbf{5 h m C}$ in ESCs, notably of key pluripotency-related mRNAs. a Reduced global 5hmC in TKO ESCs, as measured by mass spectrometry. Data are means \pm SEM ( $n=3$ independent experiments, one-tailed Student's $t$-test). Source data are provided as a Source Data File. b Decreased $5 \mathrm{hmC}$ at many peaks in TKO ESCs. Left: Box plot showing a significant difference in the normalized number of $5 \mathrm{hmC}$ reads between WT and TKO ESCs ( $n=3$ independent experiments, one-tailed Student's $t$-test). In the box plot, the boxes represent the interquartile range of the records, and the lines across the boxes indicate the median value of the records. The whiskers indicate the highest and lowest values among the records that are no more than 1.5 times greater than the interquartile range. The range between notches represents the 95\% confidence interval. Right: Quadrant chart showing differential $5 \mathrm{hmC}$ peaks in WT vs TKO ESCs. c Tet-mediated $5 \mathrm{hmC}$ marking of core pluripotency transcripts. Pie chart highlighting the percentage of transcripts whose $5 \mathrm{hmC}$ marking appears reduced, 52 of which are known to be involved in pluripotency. d Exemplative hMeRIP-Seq profiles of Eed and Dab1 in TKO vs WT ESCs (IGV tracks) (red frame shows peak location). e Top sequence motif identified at the centers of $5 \mathrm{hmC}$ peaks reduced in WT vs TKO ESCs (E-value $<3.9 \mathrm{e}-086)$. f Non-random distribution of Tet-mediated 5hmC marking. Bar chart showing, in WT and TKO ESCs, distinct distributions of $5 \mathrm{hmC}$ peaks among types of structural elements within transcripts. 
a

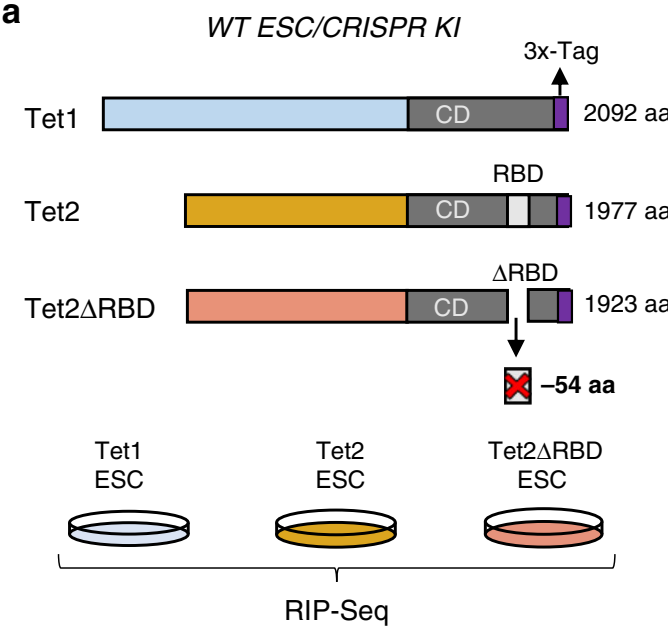

C

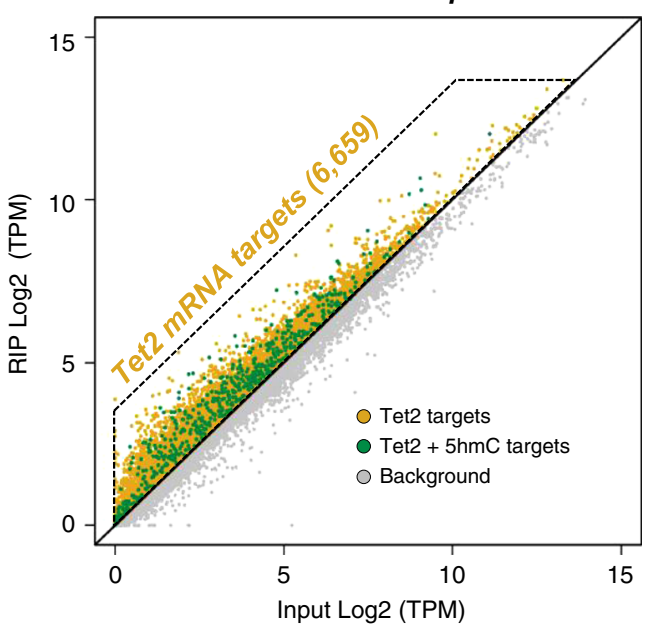

e

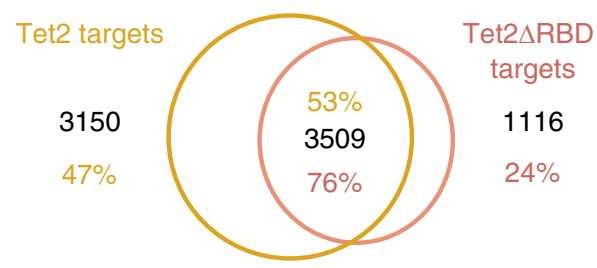

$\mathbf{f}$

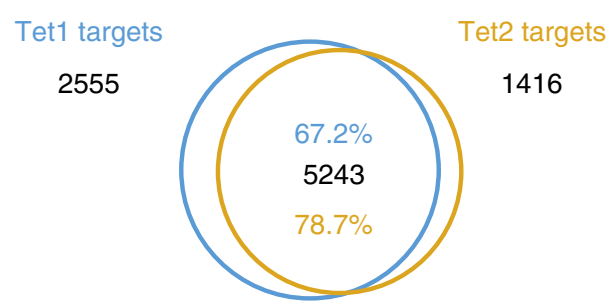

b

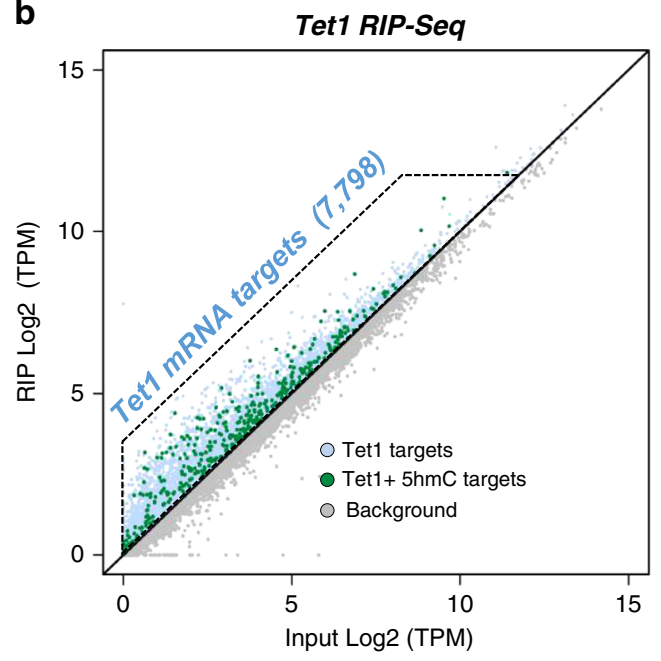

d

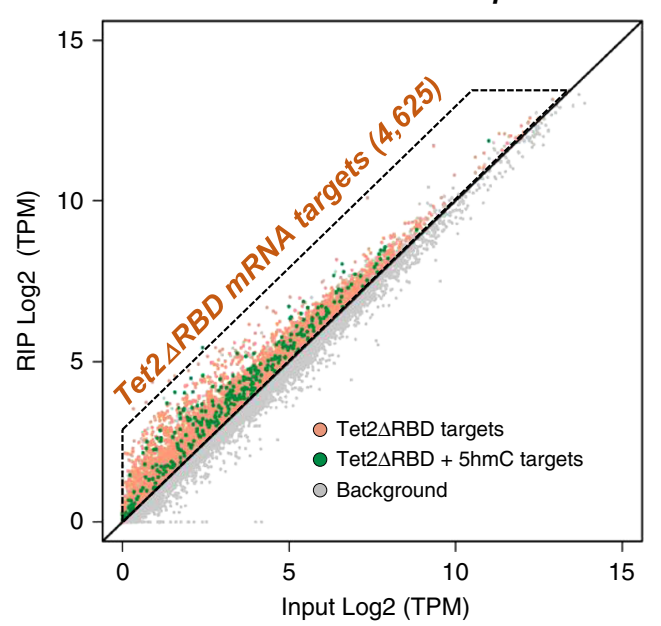

g

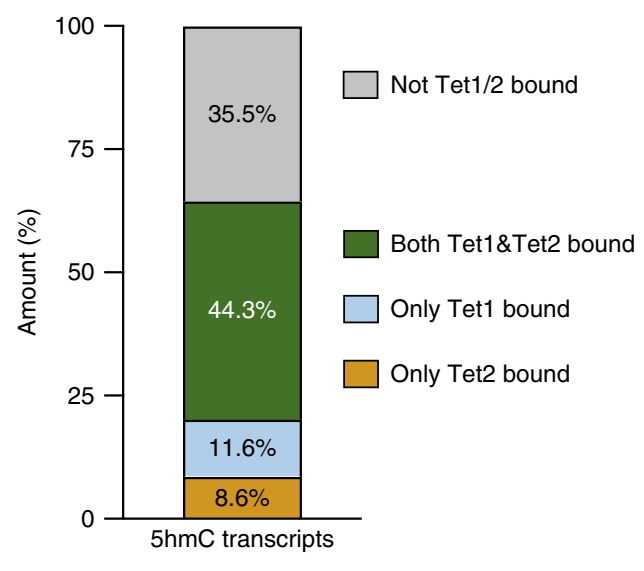

Fig. 3 Tet1 and Tet2 bind to specific mRNAs within the transcriptome. a Diagram illustrating CRISPR-mediated tagging of endogenous Tet1, Tet2, and Tet2 $\triangle$ RBD proteins (CRISPR KI) and RIP-Seq experiment design. b Many mRNAs are bound by Tet1. Scatterplot showing the log-fold enrichment over input after Tet1-bound RNA immunoprecipitation. c RNA-binding targets of Tet2. Scatterplot showing the log-fold enrichment over input after Tet2-bound RNA immunoprecipitation. d Tet2 RBD is involved in mRNA targeting. Scatterplot showing the log-fold enrichment over input after Tet2 $\triangle$ RBD-bound RNA immunoprecipitation. e Tet2 binding to many RNAs depends on Tet2 RBD. Venn diagram showing the overlap between Tet2- and Tet2 $\triangle$ RBD-bound RNA targets identified by RIP-Seq. f Many common Tet1 and Tet2 targets. Venn diagram showing the overlap between Tet1- and Tet2-bound RNA targets identified by RIP-Seq. $\mathbf{g}$ 5hmC targets are often bound by both Tet1 and Tet2. Stacked bar chart showing the overlap between $5 \mathrm{hmC}$-containing transcripts and RNA targets bound by both Tet1 and Tet2, only Tet1, or only Tet2. All RIP-Seq were performed at least in biological duplicate. 
a

hMeRIP-Seq

WT ESC/CRISPR KI Tet2 WT or $\triangle R B D$

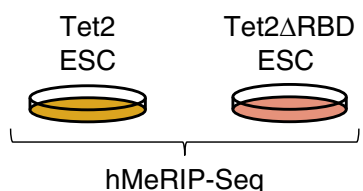

C

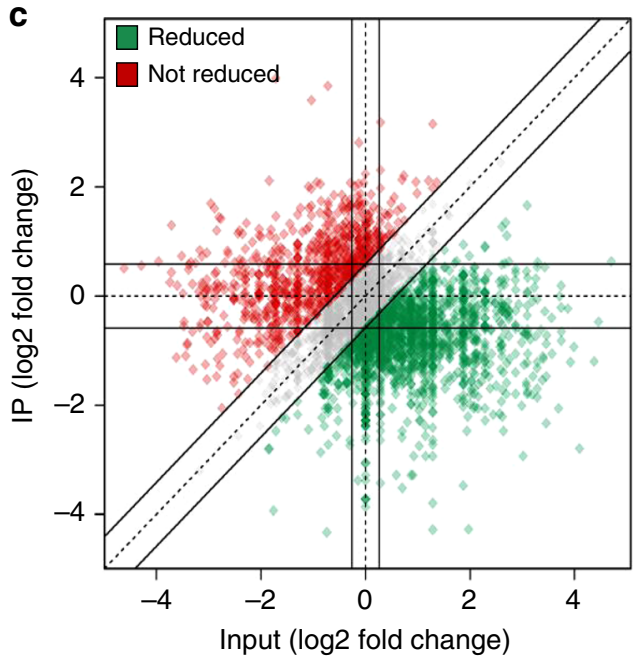

b

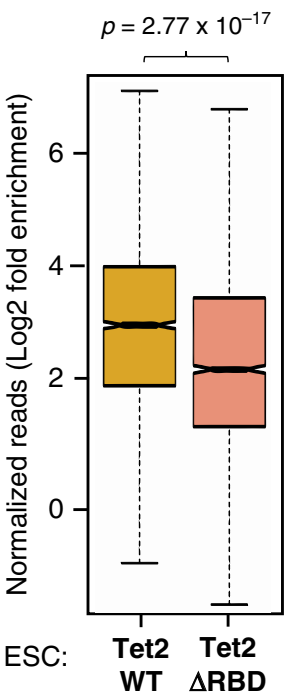

d

Differential $5 \mathrm{hmC}$ peaks

(Tet2 $\triangle$ RBD vs Tet2 WT)

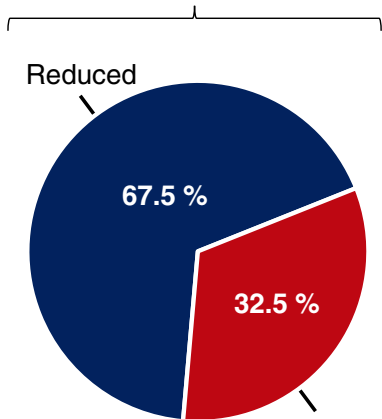

Not reduced

Fig. 4 Tet2-mediated RNA hydroxymethylation depends, at least in part, on Tet2 RBD. a Scheme illustrating the hMeRIP experimental design using CRISPR Tet2WT KI and CRISPR $\triangle$ RBD KI ESCs. b Box plot showing a significant difference in the normalized number of $5 \mathrm{hmC}$ reads between Tet2WT and Tet2 $\triangle$ RBD ESCs ( $n=3$ independent experiments, one-tailed Student's $t$-test). In the box plot, the boxes represent the interquartile range of the records, and the lines across the boxes indicate the median value of the records. The whiskers indicate the highest and lowest values among the records that are no more than 1.5 times greater than the interquartile range. The range between notches represents the 95\% confidence interval. c Quadrant chart showing differential $5 \mathrm{hmC}$ peaks in CRISPR Tet2WT KI vs CRISPR Tet2 $\triangle$ RBD KI ESCs. d Pie chart highlighting the percentage of transcripts whose $5 \mathrm{hmC}$ level is reduced in Tet2 $\triangle \mathrm{RBD}$ - compared to Tet2WT-producing cells.

among which many pluripotency-related transcripts, and that this interaction is characterized by a defined consensus site and topology.

Tet2-mediated RNA hydroxymethylation depends partially on its RBD. Having found that the Tet2 RBD contributes to Tet2 targeting and binding to transcripts (cf. Fig. $3 \mathrm{c}-\mathrm{e}$ ), we evaluated to what extent this domain is required for Tet2-mediated RNA hydroxymethylation. To this end, we performed hMeRIP-Seq with CRISPR/Cas9 knock-in ESCs for Tet2WT and TET2 $\triangle$ RBD (Fig. 4a). As depicted in Fig. 4b-d (and Supplementary Data 6), we observed a significant decrease $(67.5 \%)$ in $5 \mathrm{hmC}$-enriched regions upon the deletion of Tet2 RBD. This shows that Tet2, at least via its RBD, contributes to hydroxymethylation of mRNAs. This is in line with our recent work showing Tet2-mediated RNA hydroxymethylation of endogenous retroviruses ${ }^{30}$.
Tet-deposited 5hmC decreases mRNA stability, notably of core pluripotency transcripts. What might be the function of Tet1/2mediated mRNA hydroxymethylation in ESCs? To answer this question, we first investigated whether $5 \mathrm{hmC}$ marking might correlate with transcript abundance. The identified 5hmCmodified transcripts were thus ranked according to their abundance. Most 5hmC-modified transcripts appeared in the middle parts of transcript abundance (Supplementary Fig. 4). This preference for transcripts showing medium abundance suggests that $5 \mathrm{hmC}$ is not simply a random modification occurring on abundant transcripts. We then wondered whether 5hmC-marked transcripts might differ from unmodified transcripts at the level of translation or RNA decay. To investigate this, we examined published genome-wide data sets for mESCs. The possible effect of $5 \mathrm{hmC}$ on translational efficiency was investigated by means of previously reported ribosome profiling (Ribo-Seq) data sets from WT ESC ${ }^{39}$. As shown in Fig. 5a, 5hmC-modified and unmodified 
a

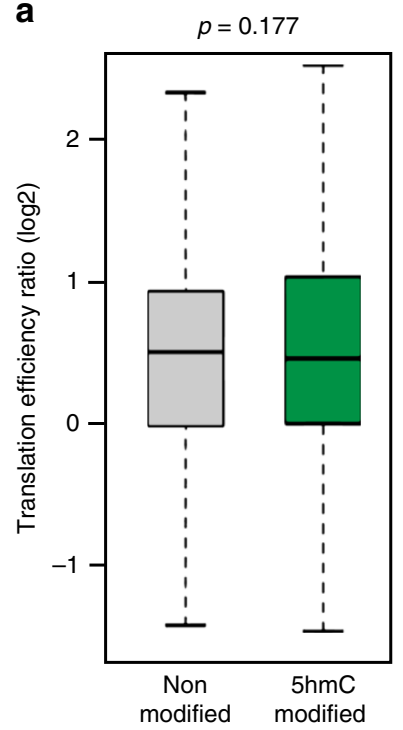

d

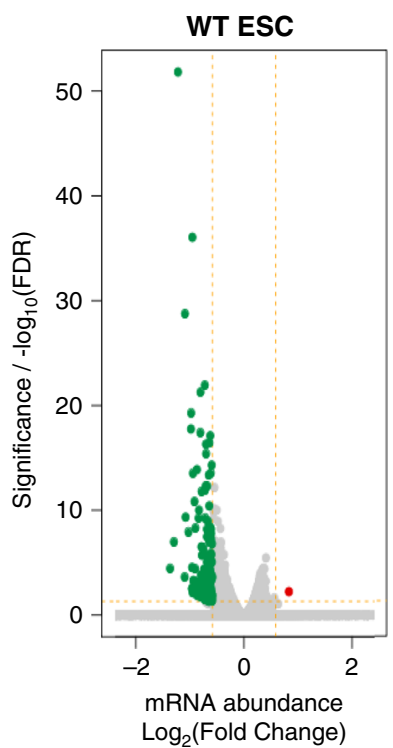

b

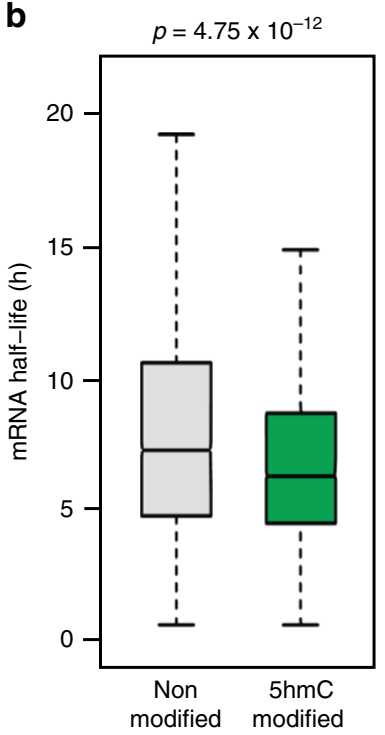

TKO ESC

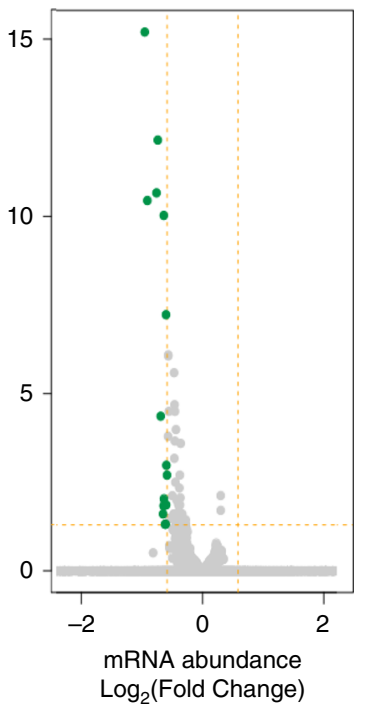

c
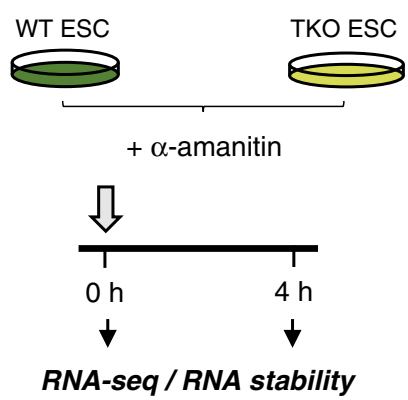

e

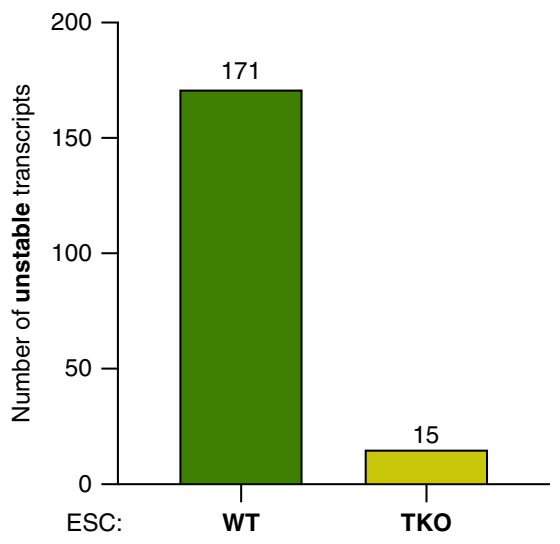

Fig. 5 Transcriptome-wide analysis of RNA stability in WT vs TKO ESCs. a $5 \mathrm{hmC}$ does not seem to impact mRNA translation. Box plot showing the translation efficiency of non-modified and 5hmC-modified transcripts in WT ESCs using our hMeRIP-Seq data $(n=3)$ and published data ${ }^{39}$ (two-tailed Wilcoxon rank-sum test, $P>0.05)$. b $5 \mathrm{hmC}$ leads to a shorter mRNA half-life. Box plot showing the difference in mRNA half-life between non-modified and $5 \mathrm{hmC}$-modified transcripts in WT ESCs using our hMeRIP-Seq data $(n=3)$ and published data 40 (two-tailed Wilcoxon rank-sum test, $P<10^{-12}$ ). In the box plot, the boxes represent the interquartile range of the records, and the lines across the boxes indicate the median value of the records. The whiskers indicate the highest and lowest values among the records that are no more than 1.5 times greater than the interquartile range. c Scheme illustrating the protocol for transcription inhibition with $\alpha$-amanitin in WT and TKO ESCs followed by RNA-Seq. Experiments were performed in biological duplicate. d Volcano plots show longer mRNA half-lives in Tet-depleted than WT ESCs. ( $P$-value corrected for multi-testing $<0.05$ and FC $>1.5$ ). e Bart chart showing a greater number of unstable transcripts in WT vs TKO ESCs, as determined by RNA-Seq at 0 and $4 \mathrm{~h}$ post $\alpha$-amanitin treatment.

transcripts showed no difference in translation efficiency. We then examined whether $5 \mathrm{hmC}$ might be associated with mRNA stability by analyzing a published data set for mRNA half-life in $\mathrm{ESCs}^{40}$. As depicted in Fig. 5b, 5hmC-marked transcripts displayed a significantly shorter mRNA half-life than unmodified transcripts $\left(P<10^{-12}\right)$. These results suggest that $5 \mathrm{hmC}$ is a chemical mark associated with transcript turnover.

To confirm the effect of $5 \mathrm{hmC}$ deposition on transcript stability, we added $\alpha$-amanitin to WT and TKO ESCs to inhibit transcription and performed RNA-Seq (Fig. 5c). As depicted in Fig. 5d, e (and Supplementary Data 7), we observed longer mRNA half-lives upon Tet depletion in TKO vs WT ESCs. These results suggest a role for Tet-mediated hydroxymethylation in mRNA stability.

To further probe the contribution of $5 \mathrm{hmC}$ in transcript stability, we produced unmodified and 5hmC-modified transcripts by in vitro transcription in the presence of $\mathrm{C}$ or $5 \mathrm{hmC}$ nucleotides and used them to transfect WT ESCs. Their abundance was measured $6 \mathrm{~h}$ and $24 \mathrm{~h}$ post-transfection in order to evaluate their relative stability (Fig. 6a). We observed after ESC transfection that in vitro $5 \mathrm{hmC}$-modified transcripts were less stable than their unmodified counterparts (Fig. 6b). These in vitro data are in good agreement with our above data showing that Tetmediated 5 hmC favors fast turnover of RNA transcripts. 
a

In vitro modified transcripts

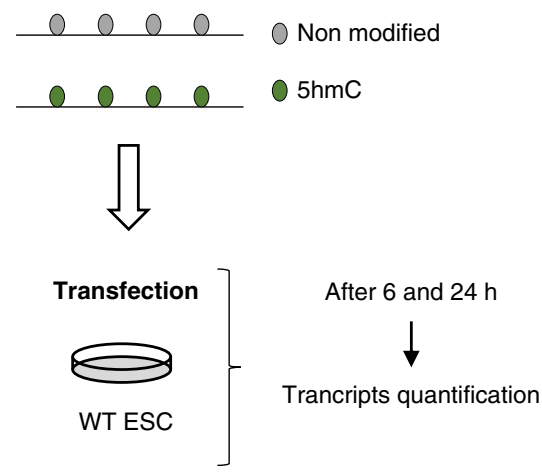

C

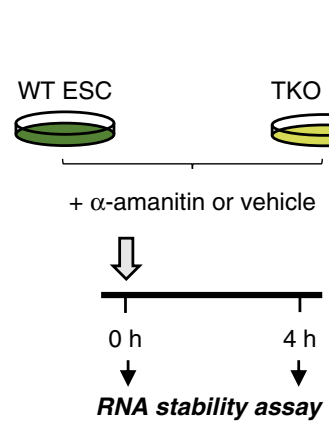

b

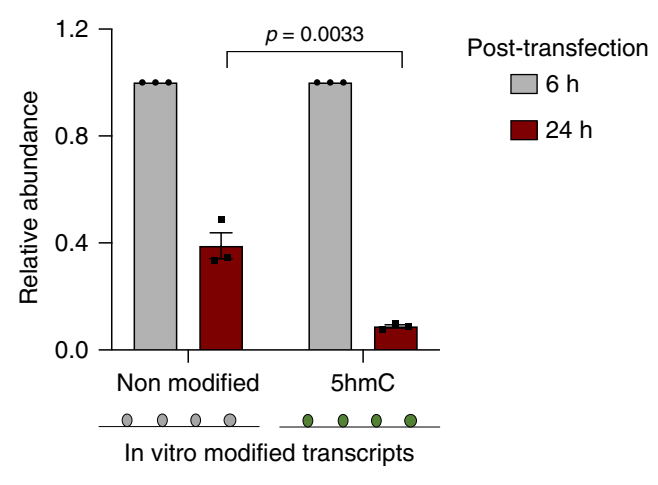

d

d $\quad$ RNA stability assay: Rescue Tet2WT/Mut in TKO ESC
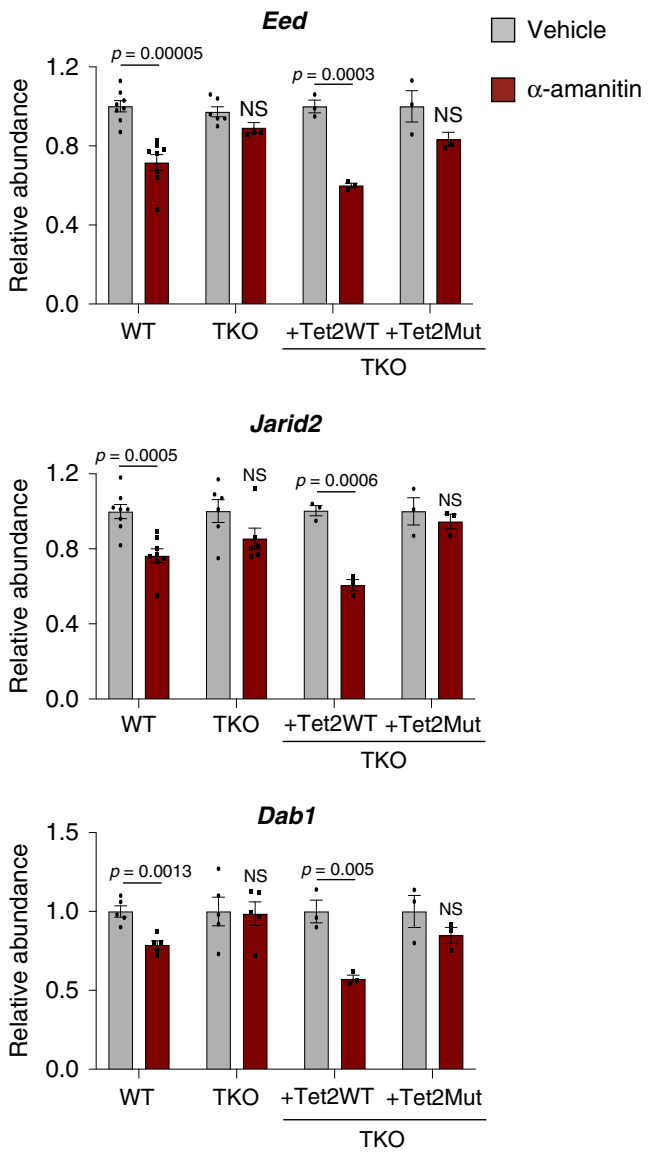

Fig. 6 Tet-mediated $5 \mathrm{hmC}$ reduces the stability of core pluripotency transcripts. a Scheme illustrating the protocol used to quantify in vitro unmodified and $5 \mathrm{hmC}$-modified transcript abundance after ESC transfection. $\mathbf{b}$ In vitro 5hmC-modified transcripts are less stable than their unmodified counterparts. Unmodified and $5 \mathrm{hmC}$-modified transcript abundances were measured by RT-qPCR at 6 and $24 \mathrm{~h}$ post-transfection. RNA levels were normalized to endogenous Gapdh and relative to the transcript level at $6 \mathrm{~h}$ post-transfection. Data are means \pm SEM ( $n=3$ independent experiments, two-tailed Student's $t$-test). c Scheme illustrating the protocol used to inhibit transcription with $\alpha$-amanitin in WT and TKO ESCs before performing stability assays to detect rescue of the destabilization process by Tet2WT or its catalytic mutant (Tet2Mut). d Tet2WT but not Tet2Mut restores destabilization of Eed, Jarid2, and Dab1 transcripts in TKO ESCs. 18S rRNA was used as an internal calibrator. Error bars indicate \pm SEM for at least three independent experiments. (Twotailed Student's $t$-test; NS not significant). Source data are provided as a Source Data File.

To validate our findings in vivo, we added $\alpha$-amanitin to WT and TKO ESCs and monitored, by qPCR, levels of key pluripotency-related mRNAs over a $4 \mathrm{~h}$ treatment period. To confirm the involvement of Tet proteins in hydroxymethylation of pluripotency-related mRNAs and the transcript-destabilizing effect of hydroxymethylation, we performed rescue experiments on TKO ESCs with Tet2WT or a Tet2 catalytic mutant (Tet2Mut) (Fig. 6c). As shown in Fig. 6d, Eed, Jarid2, and Dab1 transcripts were significantly less stable in WT ESCs than in TKO ESCs. Moreover, we found wild-type Tet2, but not Tet2Mut, to rescue 
the mechanism that destabilizes these transcripts in WT ESCs. An additional example and the $18 S$ RNA control are shown in Supplementary Fig. 5a.

Finally, we extended our observations on the above pluripotencyrelated mRNA stability assay by using CRISPR/Cas9 knock-in ESCs for Tet2WT or Tet2 $\triangle \mathrm{RBD}$. We found Tet2WT, but not Tet $2 \Delta \mathrm{RBD}$, to decrease the abundance of pluripotency transcripts (Supplementary Fig. 5b).

Overall, these results suggest that $5 \mathrm{hmC}$ deposition on pluripotency-related transcripts facilitates their degradation, which depends, at least in part, on Tet2 catalytic activity and on the RBD.

\section{Discussion}

Here we provide evidence of an additional level of regulation of the ESC self-renewal network: RNA hydroxymethylation by Tet enzymes. Our data support a stepwise working model whereby $5 \mathrm{hmC}$ mRNA modification acts as an essential regulatory layer to safeguard efficient, timely, authentic downregulation of lineagespecific genes. In this manner, $5 \mathrm{hmC}$ can promote a fast response to external cues during cell differentiation (Fig. 7). Specifically, it is well known that a gene expression program in ESCs allows them to self-renew, yet they remain poised to differentiate into all cell types in response to developmental cues. For this, key cell fate determinants need to be expressed to appropriate levels, ensuring that lineage-specific genes are adequately repressed, thus ensuring orderly differentiation of $\mathrm{ESC}^{41}$. For example, should pluripotency factors be too highly expressed, this would lead to strong silencing of lineage-commitment genes, with cells remaining in the pluripotent state. On the basis of our data, we propose a model whereby $5 \mathrm{hmC}$ marks key ESC fate determinants to limit their levels and ensure their continuous degradation. Concretely, $5 \mathrm{hmC}$ would contribute to controlling the abundance of pluripotency-associated factors (such as Eed or Jarid2), so that they are expressed at appropriate levels (not too high, not too low). This would ensure adequate repression of lineage-specific factors and critically prepare ESCs to rapidly respond to differentiation stimuli (Fig. 7).

Along with the well-described role of DNA hydroxymethylation by Tets in ES cells ${ }^{19,42,43}$, our present findings must now be taken into account if one is to understand fully the functional importance of Tets in pluripotency and lineage commitment. Future work should address how Tet enzymes discriminate between DNA and RNA substrates for hydroxymethylation. Elements that might guide Tets to specific substrates include Tetinteracting proteins $s^{30,42}$, protein $O$-glycosylation marks ${ }^{44,45}$, secondary structure, and structural changes, among other possibilities.

Regarding which Tets are responsible, in ESCs, for $5 \mathrm{hmC}$ marking of RNAs, our study suggests that Tet3 is not involved but that both Tet 1 and Tet 2 contribute similarly to RNA m5C oxidation and have redundant functions. Future analyses will be needed to decipher the mechanisms through which Tet1 and Tet2 can substitute for one another in RNA m5C oxidation. Our findings do not exclude the involvement of Tet3 in other cell contexts. Worth adding is our observation that Tet enzymes are only partly responsible for depositing $5 \mathrm{hmC}$ in mRNA, consistently with earlier reports ${ }^{24}$. Although we cannot exclude the possibility that other chemical processes besides ROS-related ones might be involved, it could be that enzymes other than Tets deposit $5 \mathrm{hmC}$ on RNA. Such enzymes would probably belong, like the Tet proteins, to the family of ferrous-ion- and $\alpha-K G$ dependent dioxygenases $\left(\mathrm{Fe}^{2+}\right.$ and 2-OG). Further study is warranted to identify additional RNA hydroxymethyltransferases.

An important finding of the present work is the identified transcriptome-wide catalog of Tet1- and Tet2-bound mRNAs. It appears that the majority of $5 \mathrm{hmC}$-modified mRNAs are bound by Tet 1 and Tet 2 , at a defined consensus site with a defined topology. We further show that a recently identified Tet2 RNAbinding domain ${ }^{38}$ is crucial for Tet2 targeting to specific transcripts and for their subsequent hydroxymethylation. An RNAbased targeting and oxidation mechanism of this type appears distinct from the reported recruitment of Tet 2 to chromatin via the RNA-binding protein $P s p c 1^{30}$. The set of Tet-interacting transcripts identified here might constitute an additional class of RNA regulons ${ }^{46}$. It is worth noting that in addition to their binding to $5 \mathrm{hmC}$-modified targets, Tet 1 and Tet 2 bind also to many unmodified transcripts. To us, this suggests the interesting possibility that besides hydroxylating mRNAs, Tet1 and Tet2 might also function independently of their catalytic activity. Such an "RNA-hydroxymethylation-independent" role would be analogous to the well-described non-catalytic action of Tet 1 and Tet2 on DNA, in which Tet proteins associate with diverse chromatinrelated machineries such as HDAC and SET1/COMPASS, involved in transcriptional repression or activation ${ }^{10}$. Tets seem likewise to have a non-catalytic action on RNA. In favor of this view, we have recently reported that a catalytic activityindependent function of Tet 2 is involved in regulating some retroviruses ${ }^{30}$. Specifically, we have shown in mouse ESCs that endogenous retrovirus (ERV) transcripts are regulated by two mechanisms: (1) post-transcriptional silencing of ERV RNAs via Tet2-mediated RNA hydroxymethylation and (2) transcriptional repression of ERVs through binding of Tet2 to RNA and concomitant recruitment of HDAC activity. Understanding the genomic characteristics that distinguish Tet1/2-bound sites that do not have $5 \mathrm{hmC}$ will require further study. Our first analyses suggest that at least some Tet1/Tet2-bound RNAs that do not have $5 \mathrm{hmC}$ display distinct DNA methylation patterns within the gene bodies of the corresponding loci.

Our study uncovers an unrecognized role of Tet-mediated RNA hydroxymethylation as a mark contributing, through mRNA destabilization, to the transcriptome flexibility required for embryonic stem cell differentiation. This role appears to be opposite to that reported for $5 \mathrm{mC}$, the $5 \mathrm{hmC}$ precursor. Among the recently reported effects of $\mathrm{m} 5 \mathrm{C}$ on mRNA fate ${ }^{47-49}$ (e.g., mRNA nuclear export, viral RNA splicing and translation), it has been shown in both physiological and pathological contexts that $\mathrm{m} 5 \mathrm{C}$ enhances mRNA stability ${ }^{50,51}$. This opposite role of $5 \mathrm{hmC}$ as compared to its precursor suggests that RNA hydroxymethylation is an important post-transcriptional modification with specific functions affecting mRNA metabolism. Accordingly, we show here that Tet-mediated hydroxymethylation can lead to downregulation and upregulation, destabilization, and splicing of modified transcripts. Considering the major roles of writers and readers in determining the regulatory roles of RNA modifications, it will be interesting in the future to characterize $5 \mathrm{hmC}$ effectors, in order to better understand the context-dependent functions of this mark, as for $\mathrm{m}^{6} \mathrm{~A}^{52}$. Besides affecting stability, it seems that $5 \mathrm{hmC}$ might also impact RNA splicing. First, we found both chromatin-associated and intronic regions of presumably unspliced nascent pre-mRNAs to be rich in $5 \mathrm{hmC}$. This suggests, as we have reported previously ${ }^{30}$, that $5 \mathrm{hmC}$ deposition might occur co-transcriptionally. Second, we found Tet-deposited $5 \mathrm{hmC}$ to correlate with a higher ratio of spliced to unspliced transcripts. While Tet-deposited $5 \mathrm{hmC}$ could have a role in splicing per se, this might also partly explain the impact of $5 \mathrm{hmC}$ on stability. In support of this, it has been reported, for example, that the half-life of the intron-less chemokine CXCL1 mRNA is shorter than that of the corresponding intron-containing control ${ }^{53}$. We propose a role of $5 \mathrm{hmC}$ as an intronic pre-mRNA modification promoting splicing and leading to a fast turnover of transcripts. The above hypothesis deserves future study. 
a

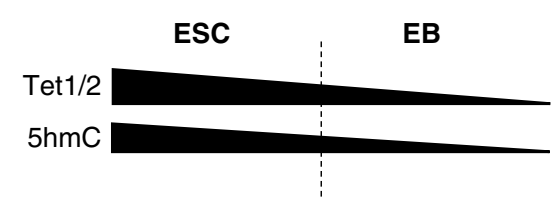

b

ESC

EB

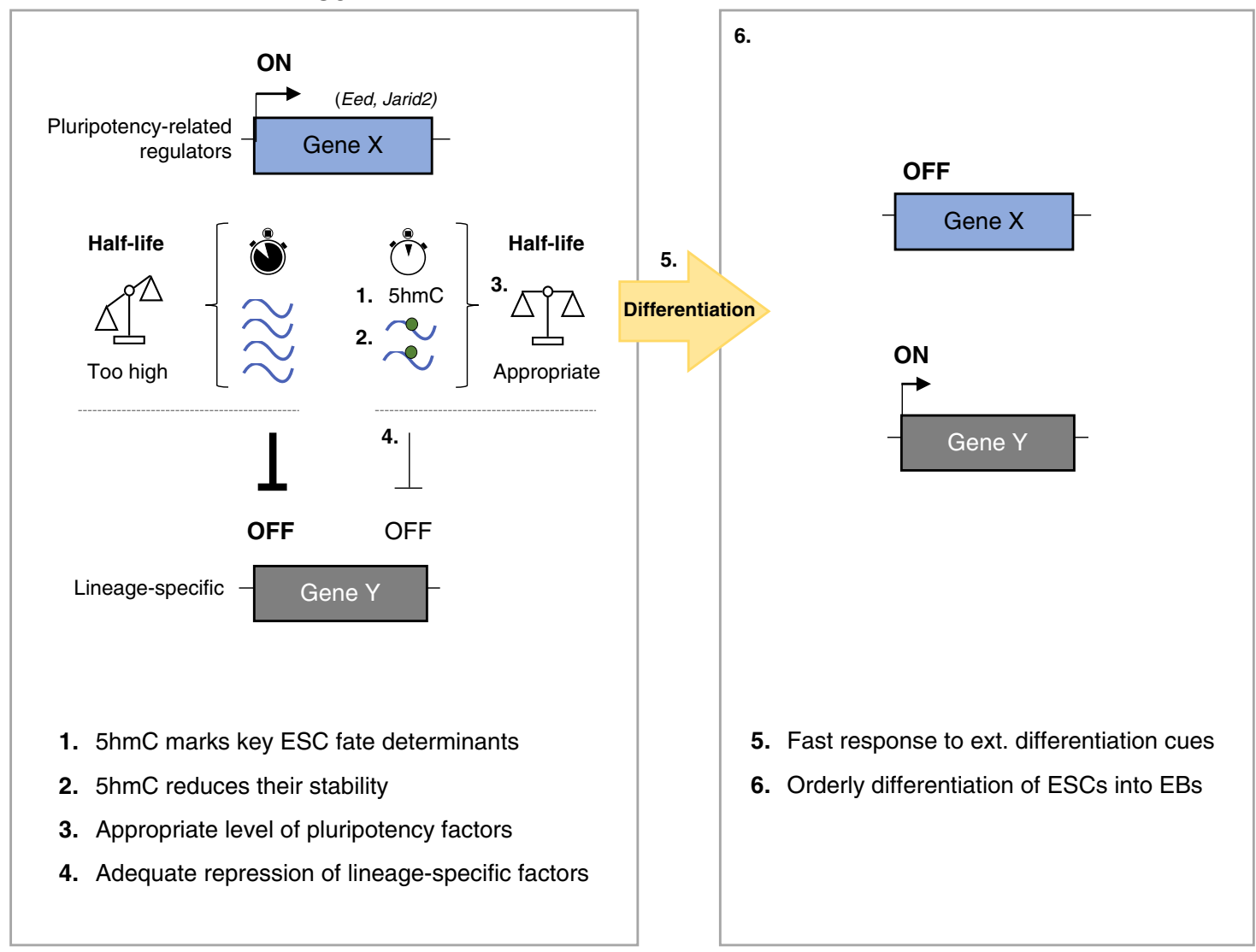

Fig. 7 Model of Tet-mediated $\mathbf{5 h m C}$ as a mark of transcriptome flexibility in ESCs. a Scheme illustrating that spontaneous ESC-to-EB differentiation leads to reduced Tet1 and Tet2 expression and to a marked decrease in $5 \mathrm{hmC}$. b $5 \mathrm{hmC}$ mRNA modification acts as an essential regulatory layer to safeguard efficient, timely, authentic downregulation of lineage-specific genes. 1. In WT ESCs, Tet1 and Tet2 hydroxymethylate many RNA transcripts, such as those encoding key pluripotency-related regulators (e.g. Eed, Jarid2). 2. 5hmC results in transcript destabilization. 3. This leads to an appropriate expression level (not too high, not too low) of these key cell fate determinants. 4. Consequently, this would ensure adequate repression of lineage-specific factors. 5. This controlled repression critically prepares ESCs to rapidly respond to external cues. 6. This stepwise model would ensure orderly differentiation of ESCs to EBs.

In conclusion, our study uncovers an unrecognized role of Tetmediated RNA hydroxymethylation as a mark contributing to the transcriptome flexibility required for embryonic stem cell differentiation. In other words, our work reveals $5 \mathrm{hmC}$ as a timely maintainer of the balance between pluripotency and lineagepriming factors, thus ensuring orderly differentiation of ESCs. Post-transcriptional RNA modifications such as $\mathrm{m}^{6} \mathrm{~A}^{31,54,55}$ and $5 \mathrm{hmC}$ should be regarded as constituting a crucial layer involved in fine-tuning gene expression in order to regulate stem cell function and developmental processes.

\section{Methods}

Cell culture. Mouse ESCs ${ }^{20,21,37}$ were grown under standard culture conditions. Briefly, cells were cultured on $0.1 \%$-gelatin-coated tissue culture plates in highglucose DMEM-containing 15\% FBS, $1 \mathrm{mM}$ sodium pyruvate, $1 \%$ non-essential amino acids, $1 \%$ glutaMAX ${ }^{\mathrm{m}}$, $100 \mathrm{U} / \mathrm{ml}$ penicillin, $100 \mu \mathrm{g} / \mathrm{ml}$ streptomycin, $0.1 \mathrm{mM}$ $\beta$-mercaptoethanol, and 1000 units/ml recombinant mouse leukemia inhibitory factor (LIF) (All reagents from Gibco, except LIF from Millipore).

Embryoid body formation. Embryoid bodies (EBs) were obtained by spontaneous differentiation of ESCs ${ }^{36}$. Briefly, ESCs were trypsinized, resuspended in ES medium without LIF, and counted with a TC20 ${ }^{\mathrm{TM}}$ Automated Cell Counter (BIORAD). Then $4 \times 10^{6}$ ESCs were seeded onto Greiner Petri dishes (Greiner) in $15 \mathrm{ml}$ ES medium without LIF. EBs were maintained in ES medium without LIF for four days before collection for further analysis.

CRISPR-Cas9 tagged Tet1, Tet2, and Tet2 $\Delta$ RBD ESCs. Mouse ESCs producing tagged Tets were generated with the CRISPR-Cas9 nuclease system ${ }^{56}$. Briefly, sgRNAs were designed to target the stop codons of Tet1 and Tet2 (for C-terminal tags) using the guidelines described in MIT's online tool (http://crispr.mit.edu)

They were cloned into the pX461 vector. Lipofectamine $e^{\text {tw }} 3000$ was used according to the manufacturer's instructions (ThermoFisher Scientific) to co-transfect ESCs with a sgRNA-containing plasmid and a template containing triple tags (Flag + $\mathrm{HA}+$ Twin-Strep) from the pINTO-N3 vector ${ }^{38}$, flanked by homologous arms for Tet1 and by homologous arms with or without RBD for Tet2. $24 \mathrm{~h}$ after 
transfection, individual ESCs were seeded into 96-well plates via serial dilution. One week later, clones were picked and analyzed for the Flag-tag by western blotting, and the CRISPR-Cas9-targeted genomic regions were PCR-amplified and sequenced in clones producing tagged Tet1, Tet2, or Tet $2 \triangle \mathrm{RBD}$. All relevant sgRNA sequences and primers are listed in Supplementary Data 8.

Cell fractionation. ESCs were washed twice with cold PBS. The cell pellet was lysed with Igepal lysis buffer (10 mM Tris $\mathrm{pH} 7.4,150 \mathrm{mM} \mathrm{NaCl}, 0.15 \%$ Igepal CA-630) and incubated on ice for $5 \mathrm{~min}$. The lysate was then gently overlaid on top of sucrose buffer $(10 \mathrm{mM}$ Tris $\mathrm{pH} 7.4,150 \mathrm{mM} \mathrm{NaCl}, 24 \%$ sucrose $)$. After centrifugation at $3500 \times g$ for $10 \mathrm{~min}$ at $4{ }^{\circ} \mathrm{C}$, the supernatant was saved for cytoplasmic RNA extraction. The pellet containing cell nuclei was briefly rinsed with cold PBS-EDTA $(0.5 \mathrm{mM})$ and resuspended in glycerol buffer $(20 \mathrm{mM}$ Tris $\mathrm{pH} 7.4$, $75 \mathrm{mM} \mathrm{NaCl}, 0.5 \mathrm{mM}$ EDTA, $50 \%$ glycerol). This was followed by the immediate addition of urea buffer (10 mM Tris pH 7.4, $300 \mathrm{mM} \mathrm{NaCl}, 7.5 \mathrm{mM} \mathrm{MgCl} 2,1 \mathrm{M}$ urea, $0.2 \mathrm{EDTA}, 1 \%$ Igepal CA-630) and incubation on ice for $2 \mathrm{~min}$. After centrifugation at $13,000 \times g$ for $2 \mathrm{~min}$ at $4^{\circ} \mathrm{C}$, the supernatant for nucleoplasmic RNA extraction was collected and the chromatin pellet was further processed with TURBO DNase followed by Proteinase K treatment before RNA extraction.

RNA and DNA extraction. Total RNA was extracted with the RNeasy Mini or RNeasy Maxi Kit (Qiagen) or with TRIzol (ThermoFisher) according to the manufacturer's instructions. Genomic DNA was eliminated by DNase I treatment. Isolated RNA was used for downstream quantitative PCR, mass spectrometry, and hMeRIP-Seq. Genomic DNA was extracted with the DNeasy Blood \& Tissue Mini Kit (Qiagen).

Reverse transcription coupled to quantitative PCR. Isolated RNA was converted to CDNA with qSCRIPT (Quanta). Gene expression was analyzed with the LightCycler 480 SYBR Green I Master mix (Roche) on the LightCycler 480 realtime PCR system (Roche). In all cases, average threshold cycles were determined from at least duplicate reactions, and gene expression levels were normalized to those of a housekeeping gene as indicated (18S rRNA, or Gapdh). The primers used in this study are shown in Supplementary Data 8.

Western blot analysis. Cells were harvested by scraping and lysed with IPH buffer containing EDTA-free Protease Inhibitor Cocktail (Roche). Cell extracts were fractionated by SDS-PAGE and transferred to PVDF membranes for immunostaining. Membranes containing the transferred proteins were blocked with 5\% $(\mathrm{w} / \mathrm{v})$ non-fat dried skimmed milk powder (Bio-rad) in PBST and then incubated overnight at $4{ }^{\circ} \mathrm{C}$ with primary antibody against Flag-tag (1:2000, Sigma \#F1804) in blocking buffer. The membranes were washed three times with PBST for $10 \mathrm{~min}$ and incubated with a 1:10,000 dilution of horseradish-peroxidase-conjugated antimouse or anti-rabbit antibodies for $1 \mathrm{~h}$. They were then washed with PBST three times and developed with the ECL system (Amersham Biosciences) according to the manufacturer's protocols. Original images for all western blots are supplied as Source Data File.

Dot blotting for $\mathbf{5 h m C}$ quantification. RNA and DNA were extracted and spotted onto a nylon membrane (GE Healthcare Hybond-N+). The membrane was dried and cross-linking was performed twice with $200,000 \mu \mathrm{J} / \mathrm{cm}^{2}$ UV. For quantification, the membrane was stained with $0.04 \%$ methylene blue in $0.5 \mathrm{M}$ sodium acetate and rinsed with PBS $+0.1 \%$ Tween-20 for $5 \mathrm{~min}$. It was then blocked in $3 \%$ $(\mathrm{w} / \mathrm{v})$ non-fat dry milk in PBS $+0.1 \%$ Tween-20 for $1 \mathrm{~h}$, transferred into a blocking solution supplemented with rat anti-5hmC monoclonal antibody (Diagenode \#MAb-633HMC) diluted 1:500 and incubated overnight at $4{ }^{\circ} \mathrm{C}$. Thereafter, the membrane was washed three times with PBS $+0.1 \%$ Tween- 20 for a total of $30 \mathrm{~min}$. It was transferred into a blocking solution supplemented with HRP-linked anti-rat IgG (Abcam \#Ab6734) diluted 1:1000, incubated for $1 \mathrm{~h}$ at room temperature, washed three times with PBS $+0.1 \%$ Tween- 20 , and developed with the ECL system (Amersham Biosciences) according to the manufacturer's protocols. ImageJ software was used for signal quantification. Original images for all dot blots are supplied as Source Data File.

LC-MS/MS for $\mathbf{5 m C}$ and $\mathbf{5 h m C}$ detection and quantification. Mass spectrometry analysis was performed as described previously ${ }^{26}$. Briefly, $3 \mu$ of $10 \times$ buffer (500 mM Tris- $\left.\mathrm{HCl}, 100 \mathrm{mM} \mathrm{NaCl}, 10 \mathrm{mM} \mathrm{MgCl}_{2}, 10 \mathrm{mM} \mathrm{ZnSO}_{4}, \mathrm{pH} 7.0\right), 2 \mu \mathrm{l}$ (180 units) of S1 nuclease, $2 \mu$ l (0.001 units) of venom phosphodiesterase I, and $1 \mu \mathrm{l}$ (30 units) of CAIP were added to $10 \mu \mathrm{g}$ of total RNA from WT ESCs and TKO ESCs (in $22 \mu \mathrm{l}$ of $\mathrm{H}_{2} \mathrm{O}$ ). The mixture $(30 \mu \mathrm{l})$ was incubated at $37^{\circ} \mathrm{C}$ for $4 \mathrm{~h}$. The resulting solution was extracted with chloroform three times. The upper aqueous phase was collected and passed through a solid-phase extraction cartridge filled with $50 \mathrm{mg}$ of sorbent of graphitized carbon black to remove the salts. The elution was then dried with nitrogen gas at $37^{\circ} \mathrm{C}$ for subsequent chemical labeling and LC-ESI-MS/MS analysis by an AB 3200 QTRAP mass spectrometer (Applied Biosystems, Foster City, CA, USA).
Vitamin $\mathbf{C}_{1} \mathbf{H}_{\mathbf{2}} \mathbf{O}_{\mathbf{2}}$, and BSO treatments. ESCs in culture were treated with $50 \mu \mathrm{M}$ Vitamin C (Sigma) for $16 \mathrm{~h}^{4}$. TKO ESCs were treated with $20 \mu \mathrm{M}$ hydrogen peroxide $^{57}\left(\mathrm{H}_{2} \mathrm{O}_{2}\right.$, Sigma) for $24 \mathrm{~h}$ or with $500 \mu \mathrm{M}$ buthionine sulfoximine ${ }^{58}$ (BSO, Sigma) for $48 \mathrm{~h}$. In each experiment, an equal volume of vehicle (water) was used as a control. Cells were collected after washing with PBS and processed for dot blotting.

In vitro transcription. In vitro transcription was performed with the MEGAscript ${ }^{\oplus}$ T7 Transcription Kits (Life Technologies) according to the manufacturer's instructions. For methylated and hydroxymethylated transcripts, ribo-CTP nucleotides were replaced in the reaction with ribo-5mCTP or ribo-5hmCTP (TriLink Biotechnologies). The DNA fragment containing TC-rich motifs was synthesized by IDT and subsequently cloned into a plasmid containing a T7 promoter. The integrity of the IVT-produced transcripts was confirmed with an Agilent 2100 Bioanalyzer and these transcripts were used later for antibody validation in hMeRIP-qPCR and in vitro stability assay.

Hydroxymethylated RNA immunoprecipitation (hMeRIP). The procedure was performed on ESCs (WT, TKO, tagged Tet2WT, and Tet2 $\triangle \mathrm{RBD}$ ) and EBs (WT) as described previously ${ }^{28}$. Briefly, $1 \mathrm{mg}$ total RNA was fragmented to an average size of 200-300 bp. It was then precipitated in ethanol, resuspended in RNase-free $\mathrm{ddH}_{2} \mathrm{O}$, and the fragmentation efficiency was checked on a Bioanalyzer RNA chip (Agilent). For immunoprecipitation, RNA fragments only or fragments spiked with $2.5 \mu \mathrm{g}$ IVT-produced transcripts containing UC-rich motifs with distinct RNA modifications $\left(\mathrm{C}, 5 \mathrm{mC}\right.$, and $5 \mathrm{hmC}$ ), were denatured by heating at $70^{\circ} \mathrm{C}$ for $5 \mathrm{~min}$ chilled on ice for $5 \mathrm{~min}$, and then incubated overnight at $4^{\circ} \mathrm{C}$ with or without $12.5 \mu \mathrm{g}$ anti-5hmC antibody (Diagenode monoclonal \#MAb-633HMC) in freshly prepared 1X IP buffer (50 mM Tris- $\mathrm{HCl} \mathrm{pH}=7.4,750 \mathrm{mM} \mathrm{NaCl}$ and $0.5 \%$ Igepal CA-630, RNasin $400 \mathrm{U} / \mathrm{ml}$ and RVC $2 \mathrm{mM}$ ) supplemented with protease inhibitors (cOmplete, Mini, EDTA-free, Roche). Samples were then incubated at $4{ }^{\circ} \mathrm{C}$ for $2.5 \mathrm{~h}$ with $60 \mu \mathrm{l}$ equilibrated Dynabeads Protein G (Life Technologies), washed three times for $5 \mathrm{~min}$ with $1 \mathrm{ml}$ IP buffer, and eluted by addition of $1 \mathrm{ml}$ TriPure Reagent (Roche). This was followed by RNA extraction according to the manufacturer's instructions. Samples were then subjected to deep sequencing and the spike-ins were analyzed by quantitative PCR (primers available in Supplementary Data 8). All hMeRIP-Seq and qPCR experiments were performed in triplicate.

RNA immunoprecipitation. RNA immunoprecipitation (RIP) was performed using Magna RIP ${ }^{\mathrm{TM}}$ RNA-Binding Protein Immunoprecipitation Kit (Millipore) following the manufacturer's instructions. Briefly, cytoplasmic extract from $\sim 1 \times$ $10^{7}$ tagged ESCs was distributed equally among samples and controls. For sample reactions, $10 \mu \mathrm{g}$ of anti-flag antibody (Sigma, \#F1804) was used for $75 \mu \mathrm{l}$ of magnetic protein $\mathrm{G}$ beads. For control reactions, $10 \mu \mathrm{g}$ of mouse IgG (Millipore, \#12 371) with no immunoreactivity was used for $75 \mu \mathrm{l}$ of magnetic protein $\mathrm{G}$ beads. After stringent washes and proteinase K digestion, immunoprecipitated RBP/RNAs (RIP) and total RNA (Input) from ESCs were subjected to downstream library preparation. All RIP-Seq experiments were performed at least in duplicate.

Library preparation and deep sequencing. 5 to $10 \mathrm{ng}$ dsDNA was subjected to 5 and $3^{\prime}$ protruding end repair, followed by the addition of non-templated adenines to the $3^{\prime}$ ends of the blunted DNA fragments, allowing ligation of Illumina multiplex adapters. The DNA fragments were then size-selected so as to remove all unligated adapters and to sequence 200-300-bp fragments. Eighteen PCR cycles were carried out to amplify the library. DNA was quantified by fluorometry with Qubit 2.0 and DNA integrity was assessed with a 2100 Bioanalyzer (Agilent). Six picomoles of the DNA library spiked with $1 \%$ PhiX viral DNA were clustered on cBot (Illumina) and then sequenced on a NextSeq500 (Illumina).

Preprocessing of sequencing data. Unless specified differently, sequencing data were preprocessed using the following steps: the raw sequencing data were first analyzed with FastQC (Andrews, 2010, https://www.bioinformatics.babraham.ac. $\mathrm{uk} /$ projects/fastqc/). Low-complexity reads were removed with the AfterQC tool ${ }^{59}$ with default parameters. To get rid of reads originating from rRNA or tRNA, the reads were mapped to mouse tRNA and rRNA sequences with Bowtie $2^{60}$. The rRNA and tRNA sequences were downloaded from https://www.ncbi.nlm.nih.gov/ nuccore using Mus musculus [organism] AND (biomol_rrna [PROP] OR biomol_trna [PROP]) as search parameters. Reads that did not map to tRNA or rRNA sequences were then further processed with Trimmomatic ${ }^{61}$ using default parameters to remove adapter sequences. The resulting fastq data were again analyzed with FastQC to ensure that no further processing was needed.

hMeRIP-sequencing analysis. Raw mouse ESCs and EBs hMeRIP-sequencing reads were preprocessed as described in the previous section. Pre-processed reads were then mapped against the mouse reference genome (mm9) with the STAR algorithm ${ }^{62}$ using the RefSeq reference transcriptome (downloaded on March 2012). $5 \mathrm{hmC}$ peak regions were identified by applying the MACS2 peak-calling tool $^{63}$ onto immunoprecipitated (IP) samples, using their input counterpart to 
estimate background noise ( $q$-value $<0.05)$. It is worth noting that the "expected genome size" MACS2 parameter was set as the sum of all transcript lengths, including both exons and introns (counting regions shared by several transcripts only once), and summit positions were identified using the MACS2 "-call-summits" option. To avoid identifying extremely large peak regions, the peaks were resized to $100 \mathrm{bp}$ on both sides of the identified summit. So-called "expected peaks" (regions with a high read count and therefore most likely to generate peaks) were also generated by applying MACS2 with the same parameters to the input only (using MACS2 background modeling). A "bedtools intersect"-based in-house script was then used to identify $5 \mathrm{hmC}$-modified regions observed in all replicate experiments ${ }^{64}$. These replicated peaks were reported as the final list of " $5 \mathrm{hmC}$ peaks" (Supplementary Data 1) (replicated "expected peaks" were also generated by the same approach). Finally, a metasample combining the mapped reads of all the replicates was generated for each condition. To obtain visual representations of local enrichment profiles, bedgraph files were generated from mapped metasample files (bam) and uploaded into the IGV tool ${ }^{65}$. For differential analysis, reads from metasamples were counted in each "replicated peak" using the FeatureCounts algorithm on IP and input samples from each condition and normalized as reads per kilobase per million (RPKM). Enrichment ratios were defined for each condition as IP over input RPKM levels. Peaks were reported as differentially marked if a fold change of at least 1.5 was observed between the enrichment ratios of the two conditions.

Motif analysis of hMeRIP peaks. To perform the motif analysis, $5 \mathrm{hmC}$ and expected peaks were associated with transcripts with "bedtools intersect" 64 on the RefSeq transcriptome. The strand of each peak was attributed to its associated transcript (unassociated peaks were ignored and peaks intersecting transcripts of both strands were duplicated). Then the peaks were extended to $250 \mathrm{bp}$ on both sides of the center and "bedtools getfasta" 64 was used to extract peak sequences in a stranded way. The meme-suite ${ }^{66}$ (http://meme-suite.org) was then used for motif analysis. A first-order Markov model was generated using the "fasta-get-markov" function on the sequences from the input sample. Then the "meme" tool was used to identify top overrepresented motifs, using the aforementioned Markov model as a background model and expected peaks as negative control peaks. The number of motifs was restricted to 10 and the MEME search window was set between 5 and 12. Finally, we used "Centrimo" to evaluate the position of the motif relatively to the peak center, and decentered motifs were excluded.

Distribution of hMeRIP peaks. The " $5 \mathrm{hmC}$ " and "expected" peaks identified by hMeRIP-Seq were annotated with the RefSeq gene annotation. Peaks were assigned to one or several transcripts and to annotated structural elements: to an exon when the peak summit was inside an annotated exon, to an intron when the peak summit was outside the exon but inside the transcript. Peaks that could not be associated with a coding gene or that could not be uniquely associated with one of these categories (e.g., ambiguous annotation due to overlapping transcripts) were left unannotated. The same rules were used to categorize peaks according to their association with coding sequences (CDS) or flanking regions ( $5^{\prime}$ UTR and $3^{\prime} \mathrm{UTR}$ ). For each transcriptomic region, the enrichment in 5hmC peaks was evaluated as the difference between the observed and expected percentages of $5 \mathrm{hmC}$ peaks in that region.

RIP sequencing analysis. Raw reads were processed as described in the "Preprocessing of sequencing data" section of this manuscript. The processed data were then mapped to the mouse genome (mm9), using the RefSeq reference transcriptome (downloaded on March 2012) and the RSEM tool ${ }^{67}$. Transcripts Per Million normalized counts (TPM) were computed from the RSEM expression counts and a pseudocount of 1 TPM was added and a transcript with higher TPM value in IP over Input was considered as Tet-enriched.

Comparison of Tet1/2-bound targets (using published data). MeDIP-Seq ${ }^{19}$ were downloaded as raw data from the SRA database (https://www.ncbi.nlm.nih gov/sra) (ERP000570)

Raw data were preprocessed as described under "Preprocessing of sequencing data" (without the rtRNA filtering step). MeDIP data were filtered for duplicate reads by means of the picard tool MarkDuplicates (http://broadinstitute.github.io/ picard/) and mapped with bowtie $2^{60}$. Peaks were identified with the MACS2 peakcalling tool ${ }^{63}$ ( $q$-value $<0.05$; expected genome size set as ' $\mathrm{mm}$ ') and summit positions were identified with the "-call-summits" MACS2 option. Annotation was finally done with a bedtools-based script (the corresponding region was counted as a gene if the peak fell between a TSS and a TTS).

Transcripts bound to Tet1 and/or 2 were intersected with the $5 \mathrm{hmC}$-containing transcripts identified in hMeRIP experiments to define $5 \mathrm{hmC}$-modified and unmodified Tet1/2-bound transcript categories. For each MeDIP sample $(n=2)$, the transcripts identified using the aforementioned annotation process were intersected with each of the two categories and the percentages of $5 \mathrm{hmC}$-marked and unmarked Tet-bound transcripts were computed. A $t$-test was then applied to compare the percentages obtained for each category.
mRNA stability and translation efficiency analyses. In order to restrict the mRNA stability and translation efficiency analyses to expressed genes, we evaluated gene expression in wild-type mouse ESC cells. For this, the Poly-A RNA-Seq data were first preprocessed as described in the "Preprocessing of sequencing data" section and mapped to the mm9 genome using STAR tool ${ }^{62}$ with the RefSeq transcriptome. Then gene expression was computed with the HTseq tool ${ }^{68}$ and converted to TPM. Genes showing more than 1 TPM were considered expressed. We then stratified the transcripts of expressed genes into $5 \mathrm{hmC}$-marked and unmarked on the basis of the presence, within the transcript, of at least one $5 \mathrm{hmC}$ peak from the hMeRIP-Seq analysis. Finally, external mRNA stability microarray data ${ }^{40}$ and ribosome-sequencing profiles ${ }^{39}$ in wild-type mouse ESCs were used to compare the mRNA half-lives and translation efficiencies of $5 \mathrm{hmC}$-modified and non-modified transcripts with a Wilcoxon test.

RNA transfection. For the in vitro stability assay, unmodified and $5 \mathrm{hmC}$-modified IVT transcripts were delivered into WT ESCs with the JetPrime polyplus reagent (Polyplus transfection) according to the manufacturer's instructions. This was followed by quantitative PCR analysis at 6 and $24 \mathrm{~h}$ post-transfection ${ }^{69}$.

$\boldsymbol{\alpha}$-Amanitin treatment. For the in vivo stability assay, $\alpha$-amanitin treatment of ESCs was performed. Briefly, WT, TKO, Tet2WT, and Tet $2 \triangle$ RBD ESCs were treated with $10 \mu \mathrm{g} / \mathrm{ml} \alpha$-amanitin (Santa Cruz) or with an equal volume of vehicle (water) as a control for 0 or $4 \mathrm{~h}$, respectively. For the rescue experiments, TKO ESCs were transfected with the Tet2FL or Tet2 catalytic mutant (Tet2Mut) ${ }^{30}$ plasmid with JetPrime polyplus reagent according to the manufacturer's instructions. They were then treated with $\alpha$-amanitin as described above. The cells were then collected after washing with PBS and processed for quantitative PCR analysis and/or RNA-Seq. For RNA-Seq, total RNA was extracted from $\alpha$-amanitin-treated cells and untreated control cells and depleted of ribosomal RNA. The RNA in this fraction was fragmented before library preparation and deep sequencing, as described above. All primers used in this study are described in Supplementary Data 8.

RNA-Seq analyses for differential expression and splicing. Sequencing reads were preprocessed as described under 'Preprocessing of sequencing data'. Preprocessed reads were then mapped against the mouse reference genome $(\mathrm{mm} 9)$ with the STAR algorithm ${ }^{62}$ using the RefSeq reference transcriptome (downloaded on March 2012). Then gene expression was computed with the HTseq tool ${ }^{68}$. Raw gene expression counts were then subjected to DESeq $2^{70}$ for normalization and analysis of differential expression analysis between control (WT and TKO ESCs) and $\alpha$-amanitin-treated cells. Similar conditions were used for splicing. IR Finder version 1.2.3 $3^{71}$ was applied to detect unspliced and spliced transcripts. Count data from processed bam files were obtained with featureCounts ${ }^{72}$ and then converted to FPKM. Genes with FPKM $>0$ were considered expressed. Only expressed genes containing intronic $5 \mathrm{hmC}$ peaks were selected and further overlapped with IR Finder output. The ratio of unspliced to spliced reads from the intersection was quantified with Bedtools ${ }^{64}$. The data were normalized to the unspliced/spliced ratio found for untreated cells at time $0 \mathrm{~h}$.

Statistics and reproducibility. Statistical analysis was performed using either the computing environment R or GraphPad Prism 7. Unless otherwise indicated, all experiments included technical replicates and were repeated at least three independent times. All statistics were evaluated by Student's $t$-test unless specified otherwise. Data and graphs are presented as means \pm SEM. The statistical significance criterion was $P<0.05$.

Reporting summary. Further information on research design is available in the Nature Research Reporting Summary linked to this article.

\section{Data availability}

The RNA-Seq, hMeRIP-Seq, and RIP-Seq data supporting the findings of this study have been deposited in the GEO repository under the accession code "GSE131902". The stemness/pluripotency signature genes were derived from the $\mathrm{ESCAPE}^{34}$ and the StemChecker ${ }^{33}$ databases and from published data ${ }^{31,32,35}$. The microarray data ${ }^{40}$ "Supplementary Table 1 [https://doi.org/10.1093/dnares/dsn030]", Ribo-Seq ${ }^{39}$ "Supplementary Table S1C [https://doi.org/10.1016/j.cell.2011.10.002]", and MeDIPSeq19 "ERP000570" supporting our study are published data. Source data are provided with this paper.

\section{Code availability}

Code supporting this study is available at a dedicated Github repository [https://github. com/martinBizet/hmC_ES].

Received: 12 July 2019; Accepted: 1 September 2020; Published online: 02 October 2020 


\section{References}

1. Kriaucionis, S. \& Heintz, N. The nuclear DNA base 5-hydroxymethylcytosine is present in Purkinje neurons and the brain. Science 324, 929-930 (2009).

2. Tahiliani, M. et al. Conversion of 5-methylcytosine to 5-hydroxymethylcytosine in mammalian DNA by MLL partner TET1. Science 324, 930-935 (2009).

3. Ito, S. et al. Tet proteins can convert 5-methylcytosine to 5-formylcytosine and 5-carboxylcytosine. Science 333, 1300-1303 (2011).

4. Blaschke, K. et al. Vitamin C induces Tet-dependent DNA demethylation and a blastocyst-like state in ES cells. Nature 500, 222-226 (2013).

5. Ko, M. et al. Impaired hydroxylation of 5-methylcytosine in myeloid cancers with mutant TET2. Nature 468, 839-843 (2010).

6. Hsu, C.-H. et al. TET1 suppresses cancer invasion by activating the tissue inhibitors of metalloproteinases. Cell Rep. 2, 568-579 (2012).

7. Lian, C. G. et al. Loss of 5-hydroxymethylcytosine is an epigenetic hallmark of melanoma. Cell 150, 1135-1146 (2012).

8. Huang, H. et al. TET1 plays an essential oncogenic role in MLL-rearranged leukemia. Proc. Natl Acad. Sci. USA 110, 11994-11999 (2013).

9. Li, Z. et al. Deletion of Tet2 in mice leads to dysregulated hematopoietic stem cells and subsequent development of myeloid malignancies. Blood 118, 4509-4518 (2011).

10. Delatte, B., Deplus, R. \& Fuks, F. Playing TETris with DNA modifications. EMBO J. 33, 1198-1211 (2014).

11. Wu, H. \& Zhang, Y. Reversing DNA methylation: mechanisms, genomics, and biological functions. Cell 156, 45-68 (2014).

12. Wu, X. \& Zhang, Y. TET-mediated active DNA demethylation: mechanism, function and beyond. Nat. Rev. Genet. 18, 517-534 (2017).

13. Wossidlo, M. et al. 5-Hydroxymethylcytosine in the mammalian zygote is linked with epigenetic reprogramming. Nat. Commun. 2, 241 (2011).

14. Inoue, A., Matoba, S. \& Zhang, Y. Transcriptional activation of transposable elements in mouse zygotes is independent of Tet3-mediated 5-methylcytosine oxidation. Cell Res. 22, 1640-1649 (2012).

15. $\mathrm{Gu}, \mathrm{T} . \mathrm{-P}$. et al. The role of Tet3 DNA dioxygenase in epigenetic reprogramming by oocytes. Nature 477, 606-610 (2011).

16. Ito, $\mathrm{S}$. et al. Role of Tet proteins in $5 \mathrm{mC}$ to $5 \mathrm{hmC}$ conversion, ES-cell selfrenewal and inner cell mass specification. Nature 466, 1129-1133 (2010).

17. $\mathrm{Wu}, \mathrm{H}$. et al. Dual functions of Tet 1 in transcriptional regulation in mouse embryonic stem cells. Nature 473, 389-393 (2011).

18. $\mathrm{Xu}$, Y. et al. Genome-wide regulation of $5 \mathrm{hmC}, 5 \mathrm{mC}$, and gene expression by Tet1 hydroxylase in mouse embryonic stem cells. Mol. Cell 42, 451-464 (2011).

19. Ficz, G. et al. Dynamic regulation of 5-hydroxymethylcytosine in mouse ES cells and during differentiation. Nature 473, 398-402 (2011).

20. Dawlaty, M. M. et al. Combined deficiency of Tet 1 and Tet 2 causes epigenetic abnormalities but is compatible with postnatal development. Dev. Cell 24, 310-323 (2013).

21. Dawlaty, M. M. et al. Loss of Tet enzymes compromises proper differentiation of embryonic stem cells. Dev. Cell 29, 102-111 (2014).

22. Koh, K. P. et al. Tetl and Tet 2 regulate 5-hydroxymethylcytosine production and cell lineage specification in mouse embryonic stem cells. Cell Stem Cell $\mathbf{8}$, 200-213 (2011).

23. Dawlaty, M. M. et al. Tet1 is dispensable for maintaining pluripotency and its loss is compatible with embryonic and postnatal development. Cell Stem Cell 9, 166-175 (2011).

24. Fu, L. et al. Tet-mediated formation of 5-hydroxymethylcytosine in RNA. J Am. Chem. Soc. 136, 11582-11585 (2014).

25. Huber, S. M. et al. Formation and abundance of 5-hydroxymethylcytosine in RNA. Chembiochem 16, 752-755 (2015).

26. Huang, W. et al. Formation and determination of the oxidation products of 5methylcytosine in RNA. Chem. Sci. 7, 5495-5502 (2016).

27. Zhang, H.-Y., Xiong, J., Qi, B.-L., Feng, Y.-Q. \& Yuan, B.-F. The existence of 5-hydroxymethylcytosine and 5-formylcytosine in both DNA and RNA in mammals. Chem. Commun. 52, 737-740 (2015).

28. Delatte, B. et al. RNA biochemistry. Transcriptome-wide distribution and function of RNA hydroxymethylcytosine. Science 351, 282-285 (2016).

29. Shen, Q. et al. Tet2 promotes pathogen infection-induced myelopoiesis through mRNA oxidation. Nature 554, 123-127 (2018).

30. Guallar, D. et al. RNA-dependent chromatin targeting of TET2 for endogenous retrovirus control in pluripotent stem cells. Nat. Genet. $\mathbf{5 0}$ 443-451 (2018).

31. Geula, S. et al. Stem cells. m6A mRNA methylation facilitates resolution of naïve pluripotency toward differentiation. Science 347, 1002-1006 (2015).

32. Fidalgo, M. et al. Zfp281 coordinates opposing functions of Tet1 and Tet 2 in pluripotent states. Cell Stem Cell 19, 355-369 (2016)

33. Pinto, J. P. et al. StemChecker: a web-based tool to discover and explore stemness signatures in gene sets. Nucleic Acids Res. 43, W72-W77 (2015).

34. $\mathrm{Xu}, \mathrm{H}$. et al. ESCAPE: database for integrating high-content published data collected from human and mouse embryonic stem cells. Database 2013, bat045 (2013).
35. Young, R. A. Control of the embryonic stem cell state. Cell 144, 940-954 (2011).

36. $\mathrm{Li}, \mathrm{T}$. et al. Critical role of Tet 3 in neural progenitor cell maintenance and terminal differentiation. Mol. Neurobiol. 51, 142-154 (2015).

37. $\mathrm{Li}, \mathrm{X}$. et al. Tet proteins influence the balance between neuroectodermal and mesodermal fate choice by inhibiting Wnt signaling. Proc. Natl Acad. Sci. USA 113, E8267-E8276 (2016).

38. He, C. et al. High-resolution mapping of RNA-binding regions in the nuclear proteome of embryonic stem cells. Mol. Cell 64, 416-430 (2016).

39. Ingolia, N. T., Lareau, L. F. \& Weissman, J. S. Ribosome profiling of mouse embryonic stem cells reveals the complexity and dynamics of mammalian proteomes. Cell 147, 789-802 (2011).

40. Sharova, L. V. et al. Database for mRNA half-life of 19977 genes obtained by DNA microarray analysis of pluripotent and differentiating mouse embryonic stem cells. DNA Res. Int. J. Rapid Publ. Rep. Genes Genomes 16, 45-58 (2009).

41. Smith, Z. D., Sindhu, C. \& Meissner, A. Molecular features of cellular reprogramming and development. Nat. Rev. Mol. Cell Biol. 17, 139-154 (2016).

42. Williams, K. et al. TET1 and hydroxymethylcytosine in transcription and DNA methylation fidelity. Nature 473, 343-348 (2011).

43. Etchegaray, J.-P. et al. The histone deacetylase SIRT6 controls embryonic stem cell fate via TET-mediated production of 5-hydroxymethylcytosine. Nat. Cell Biol. 17, 545-557 (2015).

44. Chen, Q., Chen, Y., Bian, C., Fujiki, R. \& Yu, X. TET2 promotes histone OGlcNAcylation during gene transcription. Nature 493, 561-564 (2013).

45. Deplus, R. et al. TET2 and TET3 regulate GlcNAcylation and H3K4 methylation through OGT and SET1/COMPASS. EMBO J. 32, 645-655 (2013).

46. Shi, Z. \& Barna, M. Translating the genome in time and space: specialized ribosomes, RNA regulons, and RNA-binding proteins. Annu. Rev. Cell Dev. Biol. 31, 31-54 (2015)

47. Amort, T. et al. Distinct 5-methylcytosine profiles in poly(A) RNA from mouse embryonic stem cells and brain. Genome Biol. 18, 1 (2017).

48. Yang, X. et al. 5-methylcytosine promotes mRNA export-NSUN2 as the methyltransferase and ALYREF as an $\mathrm{m} 5 \mathrm{C}$ reader. Cell Res. 27, 606-625 (2017).

49. Courtney, D. G. et al. Epitranscriptomic addition of $\mathrm{m} 5 \mathrm{C}$ to HIV-1 transcripts regulates viral gene expression. Cell Host Microbe 26, 217-227.e6 (2019)

50. Yang, Y. et al. RNA 5-methylcytosine facilitates the maternal-to-zygotic transition by preventing maternal mRNA decay. Mol. Cell 75, 1188-1202.e11 (2019).

51. Chen, X. et al. 5-methylcytosine promotes pathogenesis of bladder cancer through stabilizing mRNAs. Nat. Cell Biol. 21, 978-990 (2019).

52. Shi, H., Wei, J. \& He, C. Where, when, and how: context-dependent functions of RNA methylation writers, readers, and erasers. Mol. Cell 74, 640-650 (2019).

53. Zhao, C. \& Hamilton, T. Introns regulate the rate of unstable mRNA decay. $J$ Biol. Chem. 282, 20230-20237 (2007).

54. Batista, P. J. et al. m(6)A RNA modification controls cell fate transition in mammalian embryonic stem cells. Cell Stem Cell 15, 707-719 (2014).

55. Wang, Y. et al. N6-methyladenosine modification destabilizes developmental regulators in embryonic stem cells. Nat. Cell Biol. 16, 191-198 (2014).

56. Ran, F. A. et al. Genome engineering using the CRISPR-Cas9 system. Nat. Protoc. 8, 2281-2308 (2013).

57. Guo, Y.-L., Chakraborty, S., Rajan, S. S., Wang, R. \& Huang, F. Effects of oxidative stress on mouse embryonic stem cell proliferation, apoptosis, senescence, and self-renewal. Stem Cells Dev. 19, 1321-1331 (2010).

58. Delatte, B. et al. Genome-wide hydroxymethylcytosine pattern changes in response to oxidative stress. Sci. Rep. 5, 1-10 (2015).

59. Chen, S. et al. AfterQC: automatic filtering, trimming, error removing and quality control for fastq data. BMC Bioinformatics 18, 80 (2017).

60. Langmead, B. \& Salzberg, S. L. Fast gapped-read alignment with Bowtie 2. Nat Methods 9, 357-359 (2012).

61. Bolger, A. M., Lohse, M. \& Usadel, B. Trimmomatic: a flexible trimmer for Illumina sequence data. Bioinformatics Oxf. Engl. 30, 2114-2120 (2014).

62. Dobin, A. et al. STAR: ultrafast universal RNA-seq aligner. Bioinformatics 29, 15-21 (2013).

63. Zhang, Y. et al. Model-based analysis of ChIP-Seq (MACS). Genome Biol. 9, R137 (2008)

64. Quinlan, A. R. \& Hall, I. M. BEDTools: a flexible suite of utilities for comparing genomic features. Bioinformatics Oxf. Engl. 26, 841-842 (2010).

65. Robinson, J. T. et al. Integrative genomics viewer. Nat. Biotechnol. 29, 24-26 (2011).

66. Bailey, T. L. et al. MEME SUITE: tools for motif discovery and searching. Nucleic Acids Res. 37, W202-W208 (2009).

67. Li, B. \& Dewey, C. N. RSEM: accurate transcript quantification from RNASeq data with or without a reference genome. BMC Bioinformatics 12, 323 (2011). 
68. Anders, S., Pyl, P. T. \& Huber, W. HTSeq-a Python framework to work with high-throughput sequencing data. Bioinformatics Oxf. Engl. 31, 166-169 (2015).

69. Wroblewska, L. et al. Mammalian synthetic circuits with RNA binding proteins for RNA-only delivery. Nat. Biotechnol. 33, 839-841 (2015).

70. Love, M. I., Huber, W. \& Anders, S. Moderated estimation of fold change and dispersion for RNA-seq data with DESeq2. Genome Biol. 15, 550 (2014).

71. Middleton, R. et al. IRFinder: assessing the impact of intron retention on mammalian gene expression. Genome Biol. 18, 51 (2017).

72. Liao, Y., Smyth, G. K. \& Shi, W. featureCounts: an efficient general purpose program for assigning sequence reads to genomic features. Bioinformatics Oxf. Engl. 30, 923-930 (2014)

\section{Acknowledgements}

We thank Prof. Rudolf Jaenisch (Cambridge, USA) for the mouse wild type, Tet1/2 double knockout (DKO), and Tet1/2/3 triple knockout (TKO) ESCs, Prof. Anjana Rao for Tet3 knockout mESCs, and Prof. Dr. Roberto Bonasio for the pINTO-N3 vector. We also thank Mathieu Defrance and Romy Chen-Min-Tao for scripts development. J.L. was supported by BELSPO and by the Belgian "Fonds de la Recherche Scientifique" (FNRS). N.R., A.P., E.B., and B.H. were supported by the FNRS, and A.L.G. by the "Télévie". N.K. S. was supported by the ULB Foundation. F.F. is a ULB Professor. F.F.'s lab was funded by grants from the FNRS and Télévie, the "Action de Recherche Concertée" (ARC) (AUWB-2018-2023 ULB-No 7), Wallon Region grants U-CAN-REST and INTREPID (1710179-WALLINOV, INTREPID RW 7787), an FNRS Welbio grant (FNRS-WELBIOCR-2017A-04 and FNRS-WELBIO-CR-2019A-04R), the ULB Foundation and the Belgian Foundation against cancer (FCC 2016-086 FAF-F/2016/872). P.J.H. is supported by the University of Chicago Medical Scientist Training Program (MSTP; NIH MSTP training grant T32GM007281). S.N. is an HHMI fellow of the Damon Runyon Cancer Research Foundation (DRG-2215-15). Research from the M.F. laboratory was supported by Spanish Agencia Estatal de Investigación co-funded by the FEDER Program of EU (BFU2016-80899-P)(AEI/FEDER, UE) and Ramón y Cajal award (RYC-2014-16779). J.J.L.W. holds a Fellowship from the Cancer Institute of New South Wales, Australia. J.J.L.W.'s lab was supported by grants from the NHMRC Australia (1128175 and 1129901) and the Cancer Council of NSW Australia (RG19-05). J.W.'s lab was funded by grants from NYSTEM (C32569GG; C32583GG) and NIH (R01GM129157; R01HD095938; R01HL146664).

\section{Author contributions}

J.L., N.R., and F.F. designed the experiments and interpreted the data. J.L., N.R., R.D., F.M., B.H., and P.P. generated tagged Tet1, Tet2, and Tet2 $\triangle$ RBD ESC lines and performed cellular RNA fractionation, in vitro transcription, BSO and $\mathrm{H}_{2} \mathrm{O}_{2}$ treatments, RIP, western blots, RT-qPCR, and dot blots. S.N. and P.J.H. helped with RIP-Seq experiments. M.B., N.K.S., G.M., and R.S. performed bioinformatics analyses. E.C. performed antibody validation, hMeRIP, and deep-sequencing experiments. E.B. and A.L.G performed vitamin $\mathrm{C}$ treatment and related dot blots. D.G., A.F.-I., J.W., and M.F. performed $\alpha$-amanitin treatments, RNA stability assays, and rescue experiments. C.M. and B.Y. performed mass spectrometry analyses. J.J.L.W. performed paired-end RNA-Seq. J.L., N.R., and A.P. prepared the figures and assembled the revised manuscript. F.F. wrote the manuscript.

\section{Competing interests}

The authors declare no competing interests.

\section{Additional information}

Supplementary information is available for this paper at https://doi.org/10.1038/s41467 020-18729-6.

Correspondence and requests for materials should be addressed to F.F.

Peer review information Nature Communications thanks the anonymous reviewer(s) for their contribution to the peer review of this work. Peer reviewer reports are available.

Reprints and permission information is available at http://www.nature.com/reprints

Publisher's note Springer Nature remains neutral with regard to jurisdictional claims in published maps and institutional affiliations.

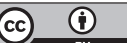

Open Access This article is licensed under a Creative Commons Attribution 4.0 International License, which permits use, sharing, adaptation, distribution and reproduction in any medium or format, as long as you give appropriate credit to the original author(s) and the source, provide a link to the Creative Commons license, and indicate if changes were made. The images or other third party material in this article are included in the article's Creative Commons license, unless indicated otherwise in a credit line to the material. If material is not included in the article's Creative Commons license and your intended use is not permitted by statutory regulation or exceeds the permitted use, you will need to obtain permission directly from the copyright holder. To view a copy of this license, visit http://creativecommons.org/ licenses/by/4.0/.

(C) The Author(s) 2020 